\title{
Quasi-disjoint pentadiagonal matrix systems for the parallelization of compact finite-difference schemes and filters
}

\author{
Jae Wook Kim* \\ Aerodynamics and Flight Mechanics Research Group, Faculty of Engineering and the Environment, University of Southampton, Southampton, SO17 1BJ, \\ United Kingdom
}

\section{A R T I C L E I N F O}

\section{Article history:}

Received 7 March 2012

Received in revised form 28 January 2013

Accepted 30 January 2013

Available online 13 February 2013

\section{Keywords:}

Parallel computing

Compact schemes

Compact filters

Computational aeroacoustics

\begin{abstract}
A B S T R A C T
This paper proposes a novel systematic approach for the parallelization of pentadiagonal compact finite-difference schemes and filters based on domain decomposition. The proposed approach allows a pentadiagonal banded matrix system to be split into quasidisjoint subsystems by using a linear-algebraic transformation technique. As a result the inversion of pentadiagonal matrices can be implemented within each subdomain in an independent manner subject to a conventional halo-exchange process. The proposed matrix transformation leads to new subdomain boundary (SB) compact schemes and filters that require three halo terms to exchange with neighboring subdomains. The internode communication overhead in the present approach is equivalent to that of standard explicit schemes and filters based on seven-point discretization stencils. The new SB compact schemes and filters demand additional arithmetic operations compared to the original serial ones. However, it is shown that the additional cost becomes sufficiently low by choosing optimal sizes of their discretization stencils. Compared to earlier published results, the proposed SB compact schemes and filters successfully reduce parallelization artifacts arising from subdomain boundaries to a level sufficiently negligible for sophisticated aeroacoustic simulations without degrading parallel efficiency. The overall performance and parallel efficiency of the proposed approach are demonstrated by stringent benchmark tests.
\end{abstract}

(c) 2013 Elsevier Inc. All rights reserved.

\section{Introduction}

Compact finite-difference schemes and filters based on implicit multi-diagonal banded matrix systems have been used for the last two decades particularly in direct numerical simulation (DNS), large eddy simulation (LES) and computational aeroacoustics (CAA) [1-12]. One of the main advantages of using them over conventional explicit ones is that one may achieve higher wavenumber performance for a given stencil size and formal order of accuracy. Currently, the vast majority of CAA/ LES/DNS activities make extensive use of parallel computing techniques benefitting from the rapid growth of modern supercomputing capabilities. However, the advantage of using the compact finite-difference schemes and filters in a parallel computing environment often comes at a significant cost, particularly when they are parallelized based on domain decomposition and message passing interface (MPI) that is currently the most common platform of parallel computing. This drawback is mainly due to their implicit nature of solving tri- or penta-diagonal banded matrix systems that demand substantial algorithmic/numerical efforts to generate parallel solutions identical to the serial counterparts.

\footnotetext{
* Tel.: +44 238059 4886; fax: +442380593058.

E-mail address: j.w.kim@soton.ac.uk
} 
Previously, some algorithmic approaches have been proposed for the parallelization of tridiagonal banded matrix systems applied to compact finite-difference schemes. They are mainly categorized into: pipelined Thomas algorithm [13,14], parallel diagonal dominant algorithm [15] and SPIKE algorithm [16]. The pipelined Thomas algorithm (PTA) provides identical results to the serial calculations at some penalty in efficiency due to processors inevitably idling at times for the completion of calculations on the other processors (mostly during the switch between forward and backward operation steps). Povitsky and Morris [14] introduced an efficient static schedule for PTA in which processors run in a time-staggered way in order to minimize the idle time. The parallel diagonal dominant (PDD) algorithm [15] is specifically designed for the solution of Toeplitz tridiagonal systems arising from compact schemes. However, it is an approximation of the original system (dropping intermediate coefficients) and has a higher computational overhead compared to PTA. Polizzi and Sameh [16] proposed an extensively generalized algorithm named SPIKE based on a four-stage procedure. The SPIKE algorithm shows more efficient and versatile performance compared to direct solvers in ScaLAPACK (an open source package for linear algebra) in parallel calculations. It can handle arbitrary bandwidth systems whereas the PTA and PDD algorithms are limited to tridiagonal ones. However, it may cost a significant computational overhead due to reordering and factorization processes required. Although these algorithmic approaches demand an increased level of complexity in computer programming, they usually provide good flexibility to be implemented in various applications.

In recent years, as an alternative to the algorithmic approaches, a boundary approximation approach was proposed to avoid the earlier drawbacks. The boundary approximation approach (BAA) employs overlapping (Gaitonde \& Visbal [7] and Sengupta et al. [17]) or halo points (Hixon [6] and Kim \& Sandberg [18]) between two neighboring subdomains and locally applies tailored boundary schemes and filters (as an approximation to the original ones) around the subdomain boundaries in order to create disjoint matrix systems that can be solved within each subdomain in a quasi-independent manner subject to a conventional message passing process. Kim and Sandberg [18] introduced a new set of wavenumber-optimized subdomain boundary schemes based on three halo points and proposed an iterative type of filtering implementation, which resulted in a reduction of numerical errors by two orders of magnitude compared to the results in [17]. They also showed that the parallel efficiency and speed-up of BAA for pentadiagonal schemes and filters could be almost comparable to those of standard explicit ones. Despite the substantial improvement achieved in [18], however, the BAA still shows parallelization artifacts in an acoustic level arising from subdomain boundaries when situated in a vortex-driven fluid flow, which raises a concern for sophisticated aeroacoustic calculations. It is indicated in [18] that the undesirable effect is essentially attributed to a local degradation in discretization performance (both dispersion and dissipation) due to the approximate boundary schemes and filters.

This paper aims to present a significantly more reliable BAA by deriving new subdomain boundary (SB) compact schemes and filters that provide almost identical dispersion and dissipation performance to the interior ones. This leads to almost artifact-free solutions from subdomain boundaries, which offers convincingly clean parallel computing environments for aeroacoustic calculations based on compact schemes/filters. The proposed BAA employs pentadiagonal platforms of fourth-order central finite-difference schemes and sixth-order central filters. A linear-algebraic transformation of the matrix systems allows deriving the new SB (subdomain boundary) compact schemes and filters that have wider discretization stencils than those of the interior ones. The extra stencils appear on the first two points from a subdomain boundary in the present pentadiagonal systems, which requires additional floating-point operations compared to the previous approach proposed in [18]. The parallel efficiency associated with the additional cost depends on the ratio between the size of the extra stencils and the size of subdomain. The present work proposes an efficient set of stencil sizes for the SB compact schemes and filters in order to minimize the extra cost without degrading the accuracy of solutions. The accuracy and efficiency of the new BAA are validated via applications to aeroacoustic benchmark tests.

The paper is organized as follows. Section 2 briefly introduces the original form of compact schemes and filters used for serial computation. Section 3 explains the formulation of the new SB compact schemes and filters associated with the linearalgebraic transformations. Section 4 suggests an efficient set of the SB compact schemes and filters with optimal stencil sizes, and examines linear stability of the combined differencing-filtering system. Section 5 shows aeroacoustic benchmark test cases to highlight the accuracy and effectiveness of the proposed approach. Finally concluding remarks are made in Section 6.

\section{Original compact finite-difference schemes and filters in serial computing}

This section introduces the original serial form of compact finite-difference schemes and filters used in the present work. They are commonly based on pentadiagonal banded matrices and seven-point stencils with suitable boundary closure formulations.

\subsection{Compact finite-difference schemes}

The present work employs fourth-order pentadiagonal compact finite-difference schemes proposed in [11] for numerical differentiation. They can be expressed in a serial form as 
Table 1

Coefficients for central compact schemes on interior points: Eq. (2.2).

\begin{tabular}{lllll}
\hline$\alpha$ & $\beta$ & $a_{1}$ & $a_{2}$ & $a_{3}$ \\
\hline 0.5862704032801503 & $9.5495335555017055 \mathrm{e}-2$ & 0.6431406736919156 & 0.2586011023495066 & $7.140953479797375 \mathrm{e}-3$ \\
\hline
\end{tabular}

Table 2

Coefficients for non-central compact schemes on physical boundary points: Eq. (2.1).

\begin{tabular}{llll}
\hline & $i=0$ & $i=1$ & $i=2$ \\
\hline$\gamma_{i 0}$ & $(\mathrm{n} / \mathrm{a})$ & $8.360703307833438 \mathrm{e}-2$ & $3.250008295108466 \mathrm{e}-2$ \\
$\gamma_{i 1}$ & $(\mathrm{n} / \mathrm{a})$ & 0.3998040493524358 \\
$\gamma_{i 2}$ & 3.912678614078549 & 2.058102869495757 & $(\mathrm{n} / \mathrm{a})$ \\
$\gamma_{i 3}$ & $(\mathrm{n} / \mathrm{a})$ & 0.9704052014790193 & 0.7719261277615860 \\
$\gamma_{i 4}$ & $(\mathrm{n} / \mathrm{a})$ & $(\mathrm{n} / \mathrm{a})$ & 0.1626635931256900 \\
$b_{i 0}$ & $(\mathrm{n} / \mathrm{a})$ & -0.3177447290722621 & -0.1219006056449124 \\
$b_{i 1}$ & -3.456878182643609 & $(\mathrm{n} / \mathrm{a})$ & -0.6301651351188667 \\
$b_{i 2}$ & 5.839043358834730 & $-2.807631929593225 \mathrm{e}-2$ & $(\mathrm{n} / \mathrm{a})$ \\
$b_{i 3}$ & 1.015886726041007 & 1.593461635747659 & 0.65211950639666084 \\
$b_{i 4}$ & -0.2246526470654333 & 0.2533027046976367 & 0.3938843551210350 \\
$b_{i 5}$ & $8.564940889936562 \mathrm{e}-2$ & $-3.619652460174756 \mathrm{e}-2$ & $1.904944407973912 \mathrm{e}-2$ \\
$b_{i 6}$ & $-1.836710059356763 \mathrm{e}-2$ & $4.080281419108407 \mathrm{e}-3$ & $-1.027260523947668 \mathrm{e}-3$ \\
\hline
\end{tabular}

$$
\left.\begin{array}{l}
\bar{f}_{i}^{\prime}+\gamma_{01} \bar{f}_{i \pm 1}^{\prime}+\gamma_{02} \bar{f}_{i \pm 2}^{\prime}= \pm \frac{1}{\Delta x} \sum_{m=0, \neq 0}^{6} b_{0 m}\left(f_{i \pm m}-f_{i}\right) \\
\gamma_{10} \bar{f}_{i}^{\prime}+\bar{f}_{i \pm 1}^{\prime}+\gamma_{12} \bar{f}_{i \pm 2}^{\prime}+\gamma_{13} \bar{f}_{i \pm 3}^{\prime}= \pm \frac{1}{\Delta x} \sum_{m=0, \neq 1}^{6} b_{1 m}\left(f_{i \pm m}-f_{i \pm 1}\right) \\
\gamma_{20} \bar{f}_{i}^{\prime}+\gamma_{21} \bar{f}_{i \pm 1}^{\prime}+\bar{f}_{i \pm 2}^{\prime}+\gamma_{23} \bar{f}_{i \pm 3}^{\prime}+\gamma_{24} \bar{f}_{i \pm 4}^{\prime}= \pm \frac{1}{\Delta x} \sum_{m=0, \neq 2}^{6} b_{2 m}\left(f_{i \pm m}-f_{i \pm 2}\right)
\end{array}\right\} \text { for } i=\left\{\begin{array}{l}
0 \\
N
\end{array}\right.
$$

where $f_{i}$ and $f_{i}^{\prime}$ represent an objective function $f(x)$ and its spatial derivative $\partial f(x) / \partial x$ respectively at a location of interest $x_{i}$. The bar "-" is used in order to distinguish numerical derivative $\left(\bar{f}^{\prime}\right)$ from the exact derivative $\left(f^{\prime}\right)$. The discretization index runs within $0 \leqslant i \leqslant N$, where $i=0$ and $i=N$ represent the domain boundaries. The upper signs in Eq. (2.1) correspond to $i=0$ (left-hand side boundary) and the lower signs to $i=N$ (right-hand side boundary). The spatial interval $\Delta x=x_{i+1}-x_{i}$ is a constant independent of the index $i$ in the computational domain where all the grid points are equally spaced. Eq. (2.2) is the main central difference scheme for interior points and Eq. (2.1) is non-central schemes for the physical boundary points. The coefficients - determined in [11] for optimal performance - are listed in Tables 1 and 2 . The resolution characteristics of the schemes are described by pseudo-wavenumber profiles (see Section 4.1 for details) in Fig. 1.

\subsection{Compact discrete filters}

Compact filters are often used in conjunction with compact finite-difference schemes in order to ensure numerical stability and remove errors arising from unresolved wavenumbers without degrading the high resolution performance of the compact schemes. The present work employs pentadiagonal compact filters proposed in [12], which can be expressed in a serial form as

$$
\begin{aligned}
& \widehat{\Delta} f_{i}+\gamma_{\mathrm{F} 01} \widehat{\Delta} f_{i \pm 1}+\gamma_{\mathrm{F} 02} \widehat{\Delta} f_{i \pm 2}=0 \\
& \gamma_{\mathrm{F} 10} \widehat{\Delta} f_{i}+\widehat{\Delta} f_{i \pm 1}+\gamma_{\mathrm{F} 12} \widehat{\Delta} f_{i \pm 2}+\gamma_{\mathrm{F} 13} \widehat{\Delta} f_{i \pm 3}=0
\end{aligned}
$$

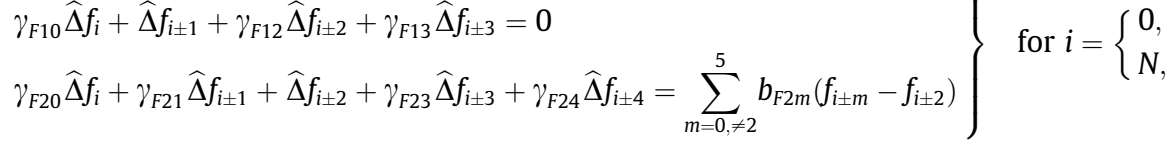

$$
\begin{aligned}
& \beta_{F} \widehat{\Delta} f_{i-2}+\alpha_{F} \widehat{\Delta} f_{i-1}+\widehat{\Delta} f_{i}+\alpha_{F} \widehat{\Delta} f_{i+1}+\beta_{F} \widehat{\Delta} f_{i+2}=\sum_{m=1}^{3} a_{F m}\left(f_{i-m}-2 f_{i}+f_{i+m}\right) \text { for } 3 \leqslant i \leqslant N-3,
\end{aligned}
$$



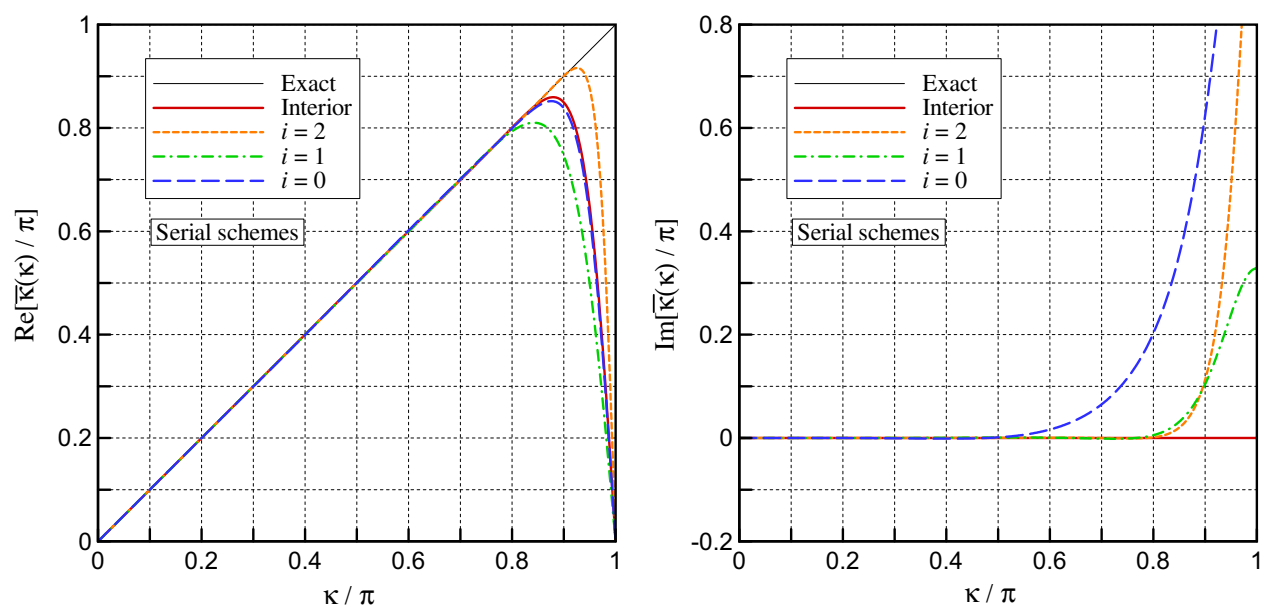

Fig. 1. Pseudo-wavenumber profiles of compact finite-difference schemes (serial mode) used in the present work: real (left) and imaginary (right) parts.

Table 3

Coefficients for central compact filters on interior points: Eq. (2.4).

\begin{tabular}{lllll}
\hline$\alpha_{F}$ & $\beta_{F}$ & $a_{F 1}$ & $a_{F 2}$ & $a_{F 3}$ \\
\hline 0.6651452077642562 & 0.1669709584471488 & $8.558206326059179 \mathrm{e}-4$ & $-3.423282530423672 \mathrm{e}-4$ & $5.705470884039454 \mathrm{e}-5$ \\
\hline
\end{tabular}

Table 4

Coefficients for non-central compact filters on physical boundary points: Eq. (2.3).

\begin{tabular}{|c|c|c|c|c|c|}
\hline Coef. & $i=0$ & $i=1$ & $i=2$ & Coef. & $i=2$ \\
\hline$\gamma_{F i 0}$ & $(\mathrm{n} / \mathrm{a})$ & 0.7311329755609861 & 0.1681680891936087 & $b_{F 20}$ & $-2.81516723801634 \mathrm{e}-4$ \\
\hline$\gamma_{F i 1}$ & 0.3412746505356879 & $(\mathrm{n} / \mathrm{a})$ & 0.6591595540319565 & $b_{F 21}$ & $1.40758361900817 \mathrm{e}-3$ \\
\hline$\gamma_{F i 2}$ & 0.2351300295562464 & 0.6689728401317021 & $(\mathrm{n} / \mathrm{a})$ & $b_{\mathrm{F} 23}$ & $2.81516723801634 \mathrm{e}-3$ \\
\hline$\gamma_{F i 3}$ & $(\mathrm{n} / \mathrm{a})$ & 0.1959510121583215 & 0.6591595540319565 & $b_{F 24}$ & $-1.40758361900817 e-3$ \\
\hline$\gamma_{F i 4}$ & $(\mathrm{n} / \mathrm{a})$ & $(\mathrm{n} / \mathrm{a})$ & 0.1681680891936087 & $b_{F 25}$ & $2.81516723801634 \mathrm{e}-4$ \\
\hline
\end{tabular}

where " $\wedge$ " represents numerically filtered quantities and $\widehat{\Delta} f_{i}=\hat{f}_{i}-f_{i}$ is the difference between the filtered and the original functions at a location of interest $x_{i}$. Again, the upper signs in Eq. (2.3) correspond to $i=0$ (left-hand side boundary) and the lower signs to $i=N$ (right-hand side boundary). Eq. (2.4) is the main central filter for interior points and Eq. (2.3) is non-central filters for the physical boundary points. The interior filter has sixth-order of accuracy and the boundary filters were derived based on a fourth-order extrapolation function in [12].

The filter coefficients in Eqs. (2.3) and (2.4) used for the present work are listed in Tables 3 and 4. The present coefficients are determined to set the cut-off wavenumber to $\kappa_{C}=0.88 \pi$ (see [12] for details) and the formulas used to determine them are also provided in Appendix A. The transfer functions of the present filters are plotted in Fig. 2 to describe the profiles of amplification rate in the wavenumber domain (see Section 4.1 for details).

\subsection{Matrix systems for compact schemes and filters}

The systems of Eqs. (2.1)-(2.4) can be expressed in a matrix-vector form:

$$
\begin{aligned}
& \mathbf{P}^{\prime}=\frac{1}{\Delta x} \mathbf{Q f} \text { for differencing, } \\
& \mathbf{P}_{F} \widehat{\Delta} \mathbf{f}=\mathbf{Q}_{\mathrm{F}} \mathbf{f} \text { for filtering, }
\end{aligned}
$$

where $\mathbf{f}, \overline{\mathbf{f}}^{\prime}$ and $\widehat{\Delta} \mathbf{f}$ are $(N+1)$-dimensional vectors representing the nodal values of objective function, numerical derivative and the filter's contribution, respectively:

$$
\mathbf{f}=\left(f_{0}, \ldots, f_{N}\right)^{\mathrm{T}}, \quad \overline{\mathbf{f}}^{\prime}=\left(\bar{f}_{0}^{\prime}, \ldots, \bar{f}_{N}^{\prime}\right)^{\mathrm{T}} \quad \& \quad \widehat{\Delta} \mathbf{f}=\left(\widehat{\Delta} f_{0}, \ldots, \widehat{\Delta} f_{N}\right)^{\mathrm{T}} .
$$

The $(N+1) \times(N+1)$ pentadiagonal matrix $\mathbf{P}$ in Eq. (2.5) is given by: 


$$
\mathbf{P}=\left(\begin{array}{ccccccccc}
1 & \gamma_{01} & \gamma_{02} & 0 & \ldots & \ldots & \ldots & \ldots & 0 \\
\gamma_{10} & 1 & \gamma_{12} & \gamma_{13} & 0 & \ldots & \ldots & \ldots & 0 \\
\gamma_{20} & \gamma_{21} & 1 & \gamma_{23} & \gamma_{24} & 0 & \ldots & \ldots & 0 \\
0 & \beta & \alpha & 1 & \alpha & \beta & 0 & \ldots & 0 \\
\vdots & \ddots & \ddots & \ddots & \ddots & \ddots & \ddots & \ddots & \vdots \\
0 & \ldots & 0 & \beta & \alpha & 1 & \alpha & \beta & 0 \\
0 & \ldots & \ldots & 0 & \gamma_{24} & \gamma_{23} & 1 & \gamma_{21} & \gamma_{20} \\
0 & \ldots & \ldots & \ldots & 0 & \gamma_{13} & \gamma_{12} & 1 & \gamma_{10} \\
0 & \ldots & \ldots & \ldots & \ldots & 0 & \gamma_{02} & \gamma_{01} & 1
\end{array}\right)
$$

and $\mathbf{P}_{F}$ in Eq. (2.6) appears the same as $\mathbf{P}$ where the coefficients contain an extra subscript " $F$ " representing the filters as defined in Eqs. (2.3) and (2.4). On the right-hand side of Eqs. (2.5) and (2.6), the $(N+1) \times(N+1)$ matrices $\mathbf{Q}$ and $\mathbf{Q}_{F}$ are as follows:

$$
\mathbf{Q}=\left(\begin{array}{ccccccccccc}
b_{00} & b_{01} & b_{02} & b_{03} & b_{04} & b_{05} & b_{06} & 0 & \ldots & \ldots & 0 \\
b_{10} & b_{11} & b_{12} & b_{13} & b_{14} & b_{15} & b_{16} & 0 & \ldots & \ldots & 0 \\
b_{20} & b_{21} & b_{22} & b_{23} & b_{24} & b_{25} & b_{26} & 0 & \ldots & \ldots & 0 \\
-a_{3} & -a_{2} & -a_{1} & 0 & a_{1} & a_{2} & a_{3} & 0 & \ldots & \ldots & 0 \\
0 & -a_{3} & -a_{2} & -a_{1} & 0 & a_{1} & a_{2} & a_{3} & 0 & \ldots & 0 \\
\vdots & \ddots & \ddots & \ddots & \ddots & \ddots & \ddots & \ddots & \ddots & \ddots & \vdots \\
0 & \ldots & 0 & -a_{3} & -a_{2} & -a_{1} & 0 & a_{1} & a_{2} & a_{3} & 0 \\
0 & \ldots & \ldots & 0 & -a_{3} & -a_{2} & -a_{1} & 0 & a_{1} & a_{2} & a_{3} \\
0 & \ldots & \ldots & 0 & -b_{26} & -b_{25} & -b_{24} & -b_{23} & -b_{22} & -b_{21} & -b_{20} \\
0 & \ldots & \ldots & 0 & -b_{16} & -b_{15} & -b_{14} & -b_{13} & -b_{12} & -b_{11} & -b_{10} \\
0 & \ldots & \ldots & 0 & -b_{06} & -b_{05} & -b_{04} & -b_{03} & -b_{02} & -b_{01} & -b_{00}
\end{array}\right),
$$

where the numbers at the diagonal of Eqs. (2.9) and (2.10) are defined as:

$$
b_{n n}=-\sum_{m=0, \neq n}^{6} b_{n m} \text { for } n=1,2 \& 3, \quad a_{F 0}=-2 \sum_{m=1}^{3} a_{F m} \quad \& \quad b_{F 22}=-\sum_{m=0, \neq 2}^{5} b_{F 2 m} .
$$

\section{Formulation of new compact schemes and filters for parallel computing}

This section presents the formulation of new compact finite-difference schemes and filters to be used on boundaries between two adjacent subdomains for parallel computing purposes based on domain decomposition and message passing. The present formulation results from linear-algebraic transformations without consideration into algorithmic techniques.

\subsection{Construction of quasi-disjoint matrix systems}

It is considered in this paper for derivation purposes that a domain is split into two subdomains from the center. In order to perform a parallel computing with the compact schemes and filters in the split subdomains, it is necessary to allow independent inversion of the pentadiagonal matrices within each subdomain. It can be achieved if some of the off-diagonal terms 

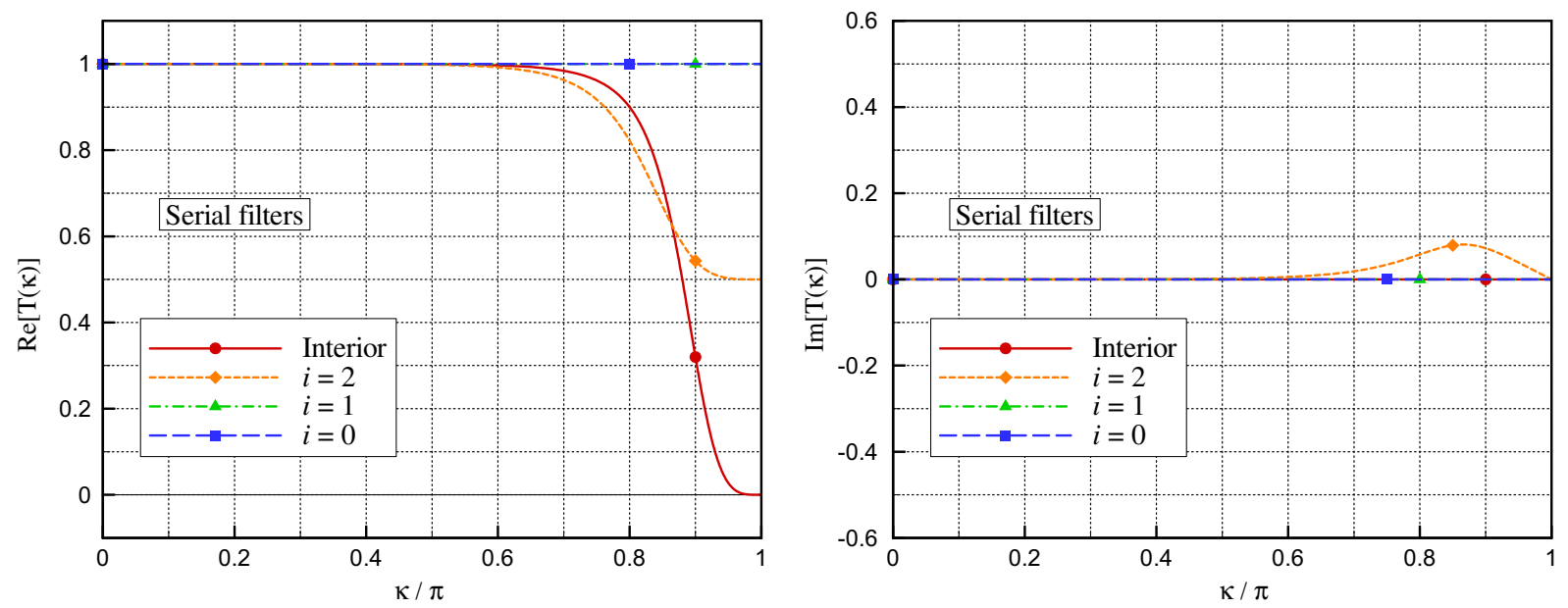

Fig. 2. Amplification rate profiles of compact filters (serial mode) used in the present work: real (left) and imaginary (right) parts.

in the matrix $\mathbf{P}$ from Eq. (2.5) are eliminated to form a modified matrix $\mathbf{R}$ that has no elements shared between the two subdomains as follows:

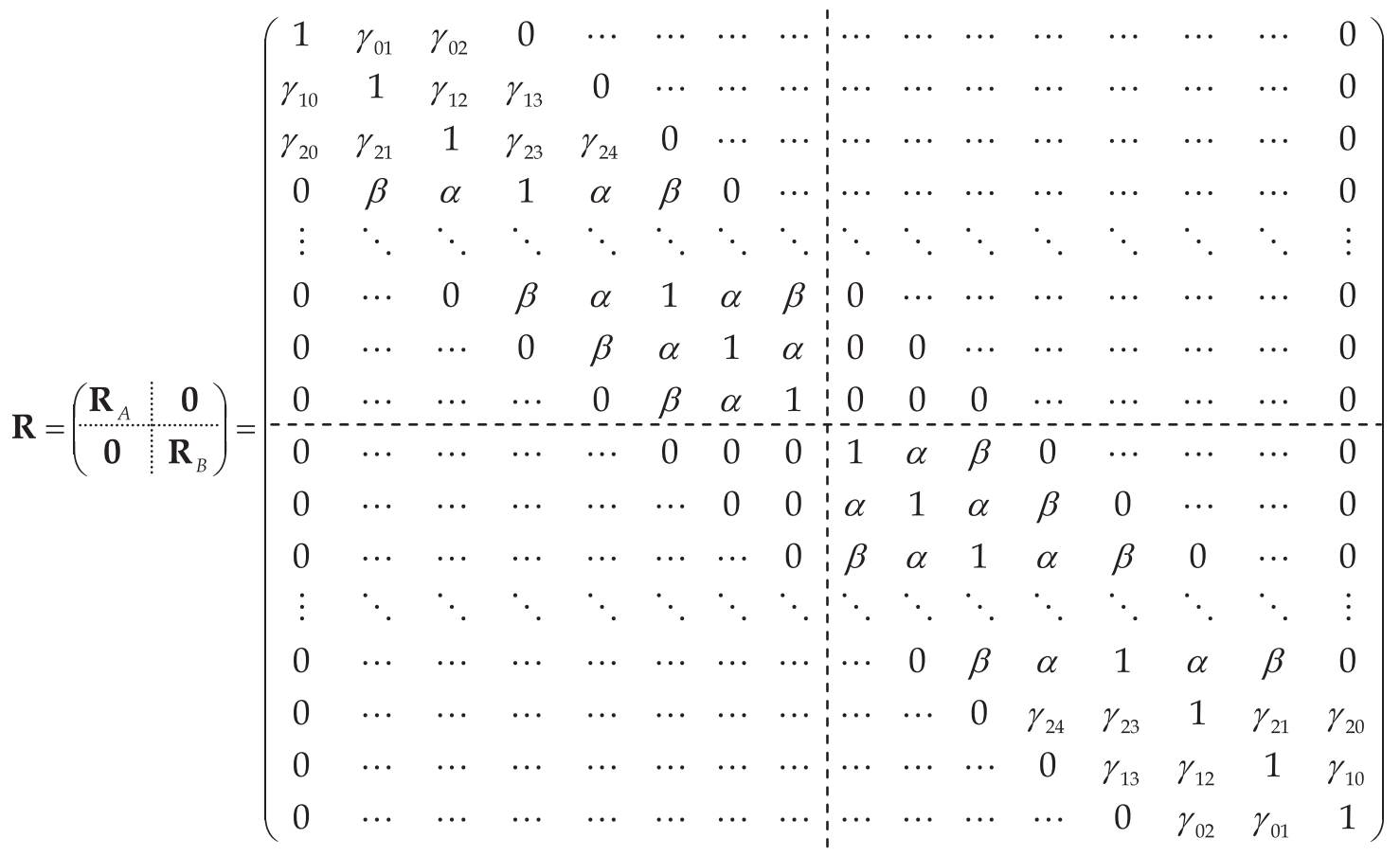

where the dashed lines represent the subdomain boundary that split the matrix into two disjoint matrices $\mathbf{R}_{A}$ and $\mathbf{R}_{B}$. The subscripts " $A$ " and " $B$ " indicate the first-half and the second-half subdomain, respectively. Likewise, $\mathbf{P}_{F}$ from Eq. (2.6) for compact filtering is modified to form:

$$
\mathbf{R}_{F}=\left(\begin{array}{c:c}
\mathbf{R}_{F A} & \mathbf{0} \\
\hdashline \mathbf{0} & \mathbf{R}_{F B}
\end{array}\right)
$$

where $\mathbf{R}_{F A}$ and $\mathbf{R}_{F B}$ appear the same as $\mathbf{R}_{A}$ and $\mathbf{R}_{B}$ in Eq. (3.1) with an extra subscript " $F$ " (representing the filters) included in the coefficients as listed in Tables 3 and 4 . It should be noted that the present approach only considers two completely separated subdomains with no overlapped points. 
For simplicity, we assume that the total number of points $N+1$ is an even number, i.e. $N+1=2(M+1)$, and the two subdomains have exactly the same number of points $M+1$ with the discretization index running from 0 to $M$. Then, the disjoint pentadiagonal matrices $\mathbf{R}_{A}$ and $\mathbf{R}_{B}$ (also $\mathbf{R}_{F A}$ and $\mathbf{R}_{F B}$ ) are of an equal size of $(M+1) \times(M+1)$ and in reversed sequence:

$$
\left.\begin{array}{l}
\left(\mathbf{R}_{B}\right)_{l, m}=\left(\mathbf{R}_{A}\right)_{M-l, M-m} \\
\left(\mathbf{R}_{F B}\right)_{l, m}=\left(\mathbf{R}_{F A}\right)_{M-l, M-m}
\end{array}\right\} \text { for }\left\{\begin{array}{c}
l=0, \cdots, M \\
m=0, \cdots, M
\end{array}\right\} \quad \text { with } M=\frac{N-1}{2}
$$

Applying $\mathbf{R}$ and $\mathbf{R}_{F}$ to the original Eqs. (2.5) and (2.6), respectively, without affecting the solutions $\overline{\mathbf{f}}^{\prime}$ and $\widehat{\Delta} \mathbf{f}$ results in the following transformation:

$$
\begin{aligned}
& \mathbf{R} \overline{\mathbf{f}}^{\prime}=\frac{1}{\Delta x} \mathbf{R} \mathbf{P}^{-1} \mathbf{Q f}=\frac{1}{\Delta x} \mathbf{S} \mathbf{f}, \\
& \mathbf{R}_{F} \widehat{\Delta} \mathbf{f}=\mathbf{R}_{F} \mathbf{P}_{F}^{-1} \mathbf{Q}_{F} \mathbf{f}=\mathbf{S}_{F} \mathbf{f} .
\end{aligned}
$$

The new matrices $\mathbf{S}=\mathbf{R} \mathbf{P}^{-1} \mathbf{Q}$ and $\mathbf{S}_{F}=\mathbf{R}_{F} \mathbf{P}_{F}^{-1} \mathbf{Q}_{F}$ on the right-hand side of Eqs. (3.4) and (3.5) can be written as:

$$
\mathbf{S}=\left(\begin{array}{l:l}
\mathbf{S}_{A} & \mathbf{T}_{B} \\
\hdashline \mathbf{T}_{A} & \mathbf{S}_{B}
\end{array}\right) \quad \& \quad \mathbf{S}_{F}=\left(\begin{array}{l:l}
\mathbf{S}_{F A} & \mathbf{T}_{F B} \\
\hdashline \mathbf{T}_{F A} & \mathbf{S}_{F B}
\end{array}\right)
$$

which leads to the following two quasi-disjoint systems for each subdomain:

$$
\begin{aligned}
& \mathbf{R}_{A} \overline{\mathbf{f}}_{A}^{\prime}=\frac{1}{\Delta x}\left(\mathbf{S}_{A} \mathbf{f}_{A}+\mathbf{T}_{B} \mathbf{f}_{B}\right) \quad \& \quad \mathbf{R}_{F A} \widehat{\Delta} \mathbf{f}_{A}=\mathbf{S}_{\mathrm{FA}} \mathbf{f}_{A}+\mathbf{T}_{F B} \mathbf{f}_{B} \text { for subdomain } A, \\
& \mathbf{R}_{B} \overline{\mathbf{f}}_{B}^{\prime}=\frac{1}{\Delta x}\left(\mathbf{S}_{B} \mathbf{f}_{B}+\mathbf{T}_{A} \mathbf{f}_{A}\right) \quad \& \quad \mathbf{R}_{F B} \widehat{\Delta} \mathbf{f}_{B}=\mathbf{S}_{F B} \mathbf{f}_{B}+\mathbf{T}_{F A} \mathbf{f}_{A} \text { for subdomain } B,
\end{aligned}
$$

where $\mathbf{f}_{A}, \overline{\mathbf{f}}_{A}^{\prime}$ and $\widehat{\Delta} \mathbf{f}_{A}$ are the first half (with the size of $M+1$ ) of the vectors in Eq. (2.7) for the subdomain $A$; and, $\mathbf{f}_{B}, \mathbf{f}_{B}^{\prime}$ and $\widehat{\Delta} \mathbf{f}_{B}$ are the second half (also with the size of $M+1$ ) for the subdomain $B$. The $(M+1) \times(M+1)$ component matrices in Eq. (3.6) are given by:

$$
\begin{aligned}
& \mathbf{S}_{A}=\left(\begin{array}{ccccccccccc}
b_{00} & b_{01} & b_{02} & b_{03} & b_{04} & b_{05} & b_{06} & 0 & \ldots & \ldots & 0 \\
b_{10} & b_{11} & b_{12} & b_{13} & b_{14} & b_{15} & b_{16} & 0 & \ldots & \ldots & 0 \\
b_{20} & b_{21} & b_{22} & b_{23} & b_{24} & b_{25} & b_{26} & 0 & \ldots & \ldots & 0 \\
-a_{3} & -a_{2} & -a_{1} & 0 & a_{1} & a_{2} & a_{3} & 0 & \ldots & \ldots & 0 \\
0 & -a_{3} & -a_{2} & -a_{1} & 0 & a_{1} & a_{2} & a_{3} & 0 & \ldots & 0 \\
\vdots & \ddots & \ddots & \ddots & \ddots & \ddots & \ddots & \ddots & \ddots & \ddots & \vdots \\
0 & \ldots & 0 & -a_{3} & -a_{2} & -a_{1} & 0 & a_{1} & a_{2} & a_{3} & 0 \\
0 & \ldots & \ldots & 0 & -a_{3} & -a_{2} & -a_{1} & 0 & a_{1} & a_{2} & a_{3} \\
0 & \ldots & \ldots & \ldots & 0 & -a_{3} & -a_{2} & -a_{1} & 0 & a_{1} & a_{2} \\
-c_{1 M} & \ldots & \ldots & \ldots & -c_{16} & -c_{15} & -c_{14} & -c_{13} & -c_{12} & -c_{11} & -c_{10} \\
-c_{0 M} & \ldots & \ldots & \ldots & -c_{06} & -c_{05} & -c_{04} & -c_{03} & -c_{02} & -c_{01} & -c_{00}
\end{array}\right), \\
& \mathbf{T}_{B}=\left(\begin{array}{cccccc}
0 & \cdots & \cdots & \cdots & \cdots & 0 \\
\vdots & \ddots & \ddots & \ddots & \ddots & \vdots \\
0 & \cdots & \cdots & \cdots & \cdots & 0 \\
a_{3} & 0 & \cdots & \cdots & \cdots & 0 \\
-c_{10}^{*} & -c_{11}^{*} & -c_{12}^{*} & -c_{13}^{*} & \cdots & -c_{1 M}^{*} \\
-c_{00}^{*} & -c_{01}^{*} & -c_{02}^{*} & -c_{03}^{*} & \cdots & -c_{0 M}^{*}
\end{array}\right), \\
& \left.\begin{array}{l}
\left(\mathbf{S}_{B}\right)_{l, m}=-\left(\mathbf{S}_{A}\right)_{M-l, M-m} \\
\left(\mathbf{T}_{A}\right)_{l, m}=-\left(\mathbf{T}_{B}\right)_{M-l, M-m}
\end{array}\right\} \text { for }\left\{\begin{array}{c}
l=0, \ldots, M \\
m=0, \ldots, M
\end{array}\right\} \text { with } M=\frac{N-1}{2},
\end{aligned}
$$




$$
\begin{aligned}
& \mathbf{S}_{F A}=\left(\begin{array}{ccccccccccc}
0 & 0 & 0 & 0 & 0 & 0 & 0 & \ldots & \ldots & \ldots & 0 \\
0 & 0 & 0 & 0 & 0 & 0 & 0 & \ldots & \ldots & \ldots & 0 \\
b_{F 20} & b_{F 21} & b_{F 22} & b_{F 23} & b_{F 24} & b_{F 25} & 0 & \ldots & \ldots & \ldots & 0 \\
a_{F 3} & a_{F 2} & a_{F 1} & a_{F 0} & a_{F 1} & a_{F 2} & a_{F 3} & 0 & \ldots & \ldots & 0 \\
0 & a_{F 3} & a_{F 2} & a_{F 1} & a_{F 0} & a_{F 1} & a_{F 2} & a_{F 3} & 0 & \ldots & 0 \\
\vdots & \ddots & \ddots & \ddots & \ddots & \ddots & \ddots & \ddots & \ddots & \ddots & \vdots \\
0 & \ldots & 0 & a_{F 3} & a_{F 2} & a_{F 1} & a_{F 0} & a_{F 1} & a_{F 2} & a_{F 3} & 0 \\
0 & \ldots & \ldots & 0 & a_{F 3} & a_{F 2} & a_{F 1} & a_{F 0} & a_{F 1} & a_{F 2} & a_{F 3} \\
0 & \ldots & \ldots & \ldots & 0 & a_{F 3} & a_{F 2} & a_{F 1} & a_{F 0} & a_{F 1} & a_{F 2} \\
c_{F 1 M} & \ldots & \ldots & \ldots & c_{F 16} & c_{F 15} & c_{F 14} & c_{F 13} & c_{F 12} & c_{F 11} & c_{F 10} \\
c_{F 0 M} & \ldots & \ldots & \ldots & c_{F 06} & c_{F 05} & c_{F 04} & c_{F 03} & c_{F 02} & c_{F 01} & c_{F 00}
\end{array}\right), \\
& \mathbf{T}_{F B}=\left(\begin{array}{cccccc}
0 & \cdots & \cdots & \cdots & \cdots & 0 \\
\vdots & \ddots & \ddots & \ddots & \ddots & \vdots \\
0 & \cdots & \cdots & \cdots & \cdots & 0 \\
a_{F 3} & 0 & \cdots & \cdots & \cdots & 0 \\
c_{F 10}^{*} & c_{F 11}^{*} & c_{F 12}^{*} & c_{F 13}^{*} & \cdots & c_{F 1 M}^{*} \\
c_{F 00}^{*} & c_{F 01}^{*} & c_{F 02}^{*} & c_{F 03}^{*} & \cdots & c_{F 0 M}^{*}
\end{array}\right) \\
& \left.\begin{array}{l}
\left(\mathbf{S}_{F B}\right)_{l, m}=\left(\mathbf{S}_{F A}\right)_{M-l, M-m} \\
\left(\mathbf{T}_{F A}\right)_{l, m}=\left(\mathbf{T}_{F B}\right)_{M-l, M-m}
\end{array}\right\} \text { for }\left\{\begin{array}{c}
l=0, \ldots, M \\
m=0, \ldots, M
\end{array}\right\} \quad \text { with } M=\frac{N-1}{2} .
\end{aligned}
$$

The reversed anti-symmetric relation between $\left(\mathbf{S}_{A}, \mathbf{T}_{A}\right)$ and $\left(\mathbf{S}_{B}, \mathbf{T}_{B}\right)$ in Eq. (3.11), and the reversed symmetric relation between $\left(\mathbf{S}_{F A}, \mathbf{T}_{F A}\right)$ and $\left(\mathbf{S}_{F B}, \mathbf{T}_{F B}\right)$ in Eq. (3.14) can only be achieved when the sizes of the two subdomains are equal. A general but more complex form of the matrices can be derived and used if required.

It can be seen from Eqs. 3.9, 3.10, 3.12 and 3.13 that only two rows from the subdomain boundary are modified by the matrix transformation outlined in Eqs. (3.4) and (3.5). All the other rows remain unchanged. The modified rows exhibit fullsized discretization stencils across both subdomains. The new coefficients $\left(c_{0 m}, c_{0 m}^{*}, c_{1 m}, c_{1 m}^{*}\right)$ and $\left(c_{F 0 m}, c_{F 0 m}^{*}, c_{F 1 m}, c_{F 1 m}^{*}\right)$ for $m=(0, \ldots, M)$ where the superscript “ $*$ " denotes coefficients for the neighboring subdomain can be obtained by using a matrix calculator depending on the size of the domain. The quasi-disjoint matrix systems introduced in Eqs. (3.7) and (3.8) provide an essential basis for the formulation of new compact schemes and filters to be used for parallel computing.

\subsection{Subdomain boundary compact schemes and filters for parallel computing}

It is not always practically sensible in a massively parallel computation, albeit possible, to derive different quasi-disjoint matrix systems on an ad hoc basis for all subdomains with different sizes. Also, computational cost is a critical issue due to the full-sized stencils involved in solving them. Therefore, this paper proposes using a universal set of subdomain boundary (SB) compact schemes and filters with a fixed (fairly small enough) size of stencils that can be used for various sizes of subdomains. This means an approximation to the original serial solution but the approximation error can be limited within a strict tolerance if the stencil sizes are carefully selected. An optimal set of the stencil sizes for both accuracy and efficiency is suggested in Section 4.

One of the main advantages in using the quasi-disjoint matrix systems introduced in Eqs. (3.7)-(3.14) is that only three halo terms (not points) are required in order to perform message passing between neighboring subdomains. The following equations can be retrieved from the quasi-disjoint matrix systems to construct new SB (subdomain boundary) compact schemes and filters employing the three halo terms, $\left(h_{0}, h_{1}, h_{2}\right)$ and $\left(h_{F 0}, h_{F 1}, h_{F 2}\right)$ :

SB compact schemes:

$$
\left.\begin{array}{l}
\bar{f}_{i}^{\prime}+\alpha \bar{f}_{i \pm 1}^{\prime}+\beta \bar{f}_{i \pm 2}^{\prime}= \pm \frac{1}{\Delta x}\left(\sum_{m=0}^{M} c_{0 m} f_{i \pm m}+h_{0}\right) \\
\alpha \bar{f}_{i}^{\prime}+\bar{f}_{i \pm 1}^{\prime}+\alpha \bar{f}_{i \pm 2}^{\prime}+\beta \bar{f}_{i \pm 3}^{\prime}= \pm \frac{1}{\Delta x}\left(\sum_{m=0}^{M} c_{1 m} f_{i \pm m}+h_{1}\right) \\
\beta \bar{f}_{i}^{\prime}+\alpha \bar{f}_{i \pm 1}^{\prime}+\bar{f}_{i \pm 2}^{\prime}+\alpha \bar{f}_{i \pm 3}^{\prime}+\beta \bar{f}_{i \pm 4}^{\prime}= \pm \frac{1}{\Delta x}\left[\sum_{m=1}^{2} a_{m}\left(f_{i \pm 2 \pm m}-f_{i \pm 2 \mp m}\right)+a_{3}\left(f_{i \pm 5}-h_{2}\right)\right.
\end{array}\right\} \text { for } i=\left\{\begin{array}{l}
0 \\
N_{S}
\end{array}\right.
$$


Table 5

Number of arithmetic operations required in implementing the present compact schemes and filters within a domain with the size of $N_{S}+1$. Abbreviations: RHS = right-hand side calculation, $\mathrm{HT}=$ halo term calculation, FS = forward substitution and BS = back substitution in a standard $L U$-decomposition routine.

\begin{tabular}{|c|c|c|c|c|}
\hline & \multicolumn{2}{|c|}{ Serial operations } & \multicolumn{2}{|c|}{ Parallel operations with proposed SB method } \\
\hline & Differencing & Filtering & Differencing & Filtering \\
\hline RHS & $8\left(N_{S}+1\right)$ & $12\left(N_{S}+1\right)$ & $8\left(N_{S}-3\right)+8(M+1)$ & $12\left(N_{S}-3\right)+8\left(M_{F}+1\right)$ \\
\hline HT & 0 & 0 & $4(2 M+1)$ & $4\left(2 M_{F}+1\right)$ \\
\hline FS & $4\left(N_{S}+1\right)$ & $4\left(N_{S}+1\right)$ & $4\left(N_{S}-1\right)+2$ & $4\left(N_{S}-1\right)+2$ \\
\hline BS & $5\left(N_{S}+1\right)$ & $5\left(N_{S}+1\right)$ & $5\left(N_{S}-1\right)+4$ & $5\left(N_{S}-1\right)+4$ \\
\hline Total & $17\left(N_{S}+1\right)$ & $21\left(N_{S}+1\right)$ & $17\left(N_{S}+1\right)+16(M-2)$ & $21\left(N_{S}+1\right)+16\left(M_{F}-3\right)$ \\
\hline
\end{tabular}

SB compact filters:

$$
\left.\begin{array}{l}
\widehat{\Delta} f_{i}+\alpha_{F} \widehat{\Delta} f_{i \pm 1}+\beta_{F} \widehat{\Delta} f_{i \pm 2}=\sum_{m=0}^{M_{F}} c_{F 0 m} f_{i \pm m}+h_{F 0} \\
\alpha_{F} \widehat{\Delta} f_{i}+\widehat{\Delta} f_{i \pm 1}+\alpha_{F} \widehat{\Delta} f_{i \pm 2}+\beta_{F} \widehat{\Delta} f_{i \pm 3}=\sum_{m=0}^{M_{F}} c_{F 1 m} f_{i \pm m}+h_{F 1} \\
\beta_{F} \widehat{\Delta} f_{i}+\alpha_{F} \widehat{\Delta} f_{i \pm 1}+\widehat{\Delta} f_{i \pm 2}+\alpha_{F} \widehat{\Delta} f_{i \pm 3}+\beta_{F} \widehat{\Delta} f_{i \pm 4}=\sum_{m=1}^{2} a_{F m}\left(f_{i \pm 2 \pm m}-2 f_{i \pm 2}+f_{i \pm 2 \mp m}\right)+a_{F 3}\left(f_{i \pm 5}-2 f_{i \pm 2}+h_{F 2}\right)
\end{array}\right\} \quad \begin{aligned}
& \text { for } i=\left\{\begin{array}{l}
0 \\
N_{S}
\end{array}\right.
\end{aligned}
$$

where $M$ and $M_{F}$ denote the stencil sizes of the SB schemes and filters, respectively, and the subdomain of interest is defined by $i \in\left[0, N_{S}\right]$ subject to conditions $N_{S} \geqslant M$ and $N_{S} \geqslant M_{F}$. It is allowed that $M_{F} \neq M$ as part of the approximation mentioned earlier. In Eqs. (3.15) and (3.16), the upper signs correspond to $i=0$ (left-hand side boundary) and the lower signs to $i=N_{S}$ (right-hand side boundary) of the subdomain. The halo terms in Eqs. (3.15) and (3.16) are given by:

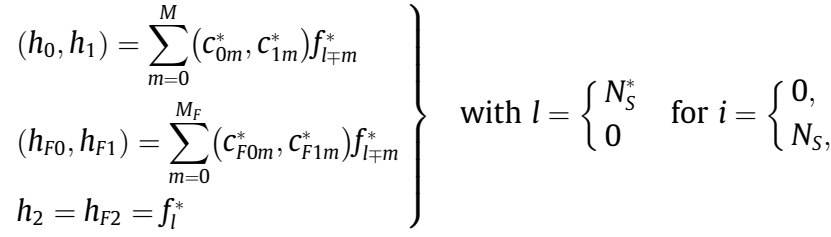

where the superscript " $*$ " and index " $l$ " denote properties in the neighboring subdomain; and, $l=0$ and $N_{S}^{*}$ represent the neighbor's boundaries in connection for message passing. It should be noted though that $l=0$ or $N_{S}^{*}$ do not point the same position to $i=0$ or $N_{S}$, since the present approach only considers completely separated subdomains with no boundary point shared or overlapped, as mentioned earlier. The coefficients $\left(c_{0 m}, c_{0 m}^{*}, c_{1 m}, c_{1 m}^{*}\right)$ for $m=(0, \ldots, M)$ and $\left(c_{F 0 m}, c_{F 0 m}^{*}, c_{F 1 m}, c_{F 1 m}^{*}\right)$ for $m=\left(0, \ldots, M_{F}\right)$ that are obtained by the procedure in Section 3.1 (for subdomains with the size of $M+1$ and $\left.M_{F}+1\right)$ are used here for any subdomains with larger sizes. Therefore, this approach is an approximate method. In this approach, $M$ and $M_{F}$
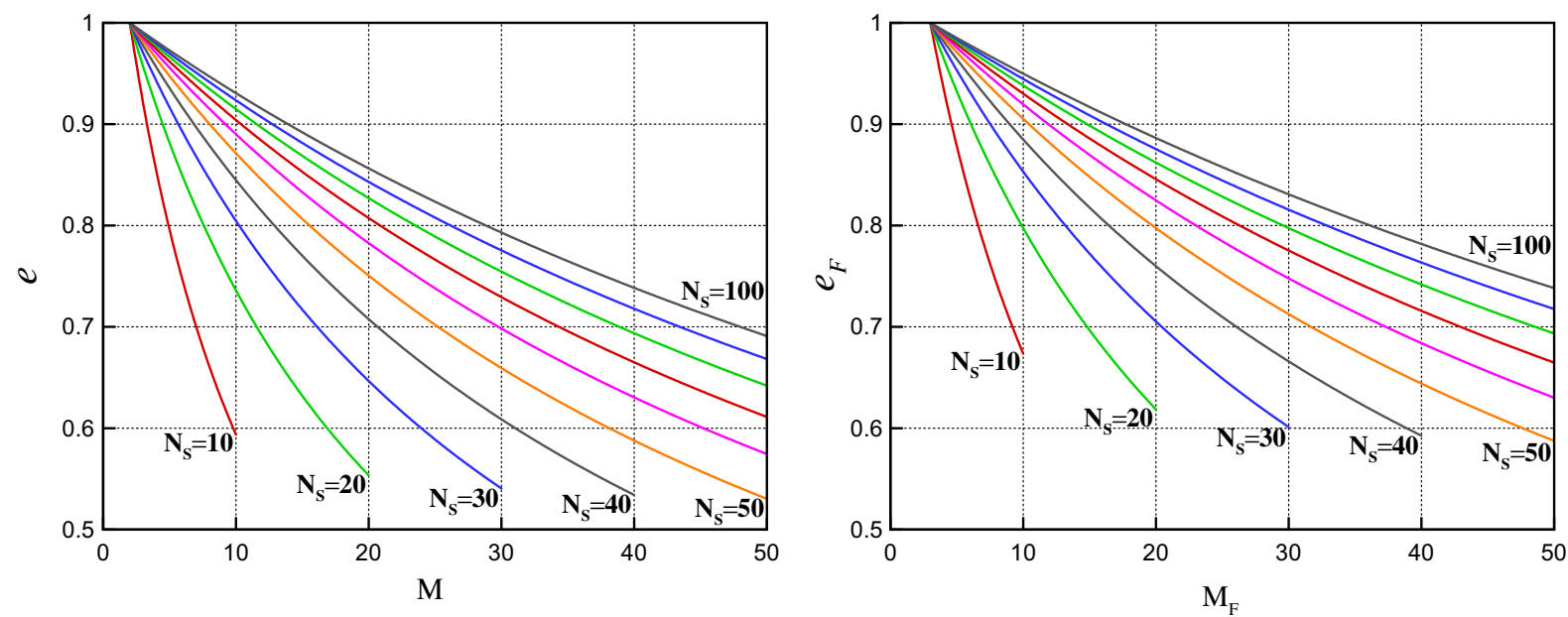

Fig. 3. Estimated parallel efficiencies of proposed SB compact schemes $(e)$ and filters $\left(e_{F}\right)$ based on Eq. (3.18). 
should be sufficiently small in order for the SB schemes and filters to fit in any reasonably sized subdomains and to keep the computational cost low.

As shown in Eqs. (3.15) and (3.16), the proposed SB compact schemes and filters rely on three halo terms and this means that their cost for internode communication is the same as those of previous ones [18] or standard explicit ones based on seven-point stencils such as DRP scheme by Tam and Webb [19] that requires three halo points. The only extra overhead involved in the present approach particularly compared to [18] is the calculation of the wider stencils ( $M$ and $M_{F}$ points) for the right-hand side of the first two equations in Eqs. (3.15) and (3.16). In Table 5, the total numbers of arithmetic operations $(+,-, \times, \div)$ required for implementing the present compact schemes and filters within a domain section with the size of $N_{S}+1$ are calculated for both serial and parallel cases to figure out the relative contribution of the extra overhead in actual calculations. This leads to an estimated parallel computing efficiency of the proposed compact schemes and filters:

$$
e=\frac{17}{17+16(M-2) /\left(N_{S}+1\right)} \quad \& \quad e_{F}=\frac{21}{21+16\left(M_{F}-3\right) /\left(N_{S}+1\right)}
$$

where subscript " $F$ " denotes filtering. The efficiency profiles are plotted in Fig. 3 as a function of $M$ and $M_{F}$ for various $N_{S}$. It is noteworthy for comparison purposes that the earlier work of [18] yields $e=1$ and $e_{F}=0.5$ since their SB schemes and filters are based on seven-point stencils (same as the interior) but the filters use double operations due to predictor-corrector iterations.

\section{Efficient stencil sizes for SB compact schemes and filters}

This section suggests an efficient set of stencil sizes for the proposed SB (subdomain boundary) compact finite-difference schemes and filters which provides high accuracy parallel solutions without a significant impact on the computational cost. Fourier analysis of errors is performed to investigate the accuracy of the parallel schemes and filters compared to the serial ones for various stencil sizes. Linear stability of the selected schemes and filters is examined as well.

\subsection{Fourier analysis of errors}

Fourier transform provides useful apparatus to investigate dispersive and dissipative behaviors of a discretization method in wavenumber domain. The use of Fourier transform for these purposes has been discussed and established through a series of publications for the last five decades as described in [1]. Mathematically, the Fourier transform of a continuous function (subject to certain conditions - not to be discussed in detail here) is defined as

$$
\tilde{f}(k)=\int_{-\infty}^{\infty} f(x) e^{-j k x} d x
$$

where $j=\sqrt{-1}, k$ is the wavenumber and the tilde represents the transformed function. With $f_{i+m}$ and $\bar{f}_{i+m}^{\prime}$ replaced by $f(x+m \Delta x)$ and $\bar{f}^{\prime}(x+m \Delta x)$, taking the Fourier transform of Eqs. (2.2), (2.4), (3.15) and (3.16) results in the following equations:

$$
\begin{aligned}
& j \bar{\kappa}\left[A_{i}(\kappa)+j B_{i}(\kappa)\right]=C_{i}(\kappa)+j D_{i}(\kappa) \text { for differencing, } \\
& {[\tilde{\hat{f}}(k)-\tilde{f}(k)]\left[A_{F i}(\kappa)+j B_{F i}(\kappa)\right]=\tilde{f}(k)\left[C_{F i}(\kappa)+j D_{F i}(\kappa)\right] \text { for filtering, }}
\end{aligned}
$$

where $\kappa=k \Delta x$ is a scaled wavenumber and $\bar{\kappa}=\bar{k} \Delta x$ is a scaled pseudo-wavenumber which deviates from the true wavenumber $(\kappa)$ due to the numerical differentiation, i.e. $\tilde{f}^{\prime}=j k \tilde{f}$ and $\tilde{f}^{\prime}=j \bar{k} \tilde{f}$. By working out Eqs. (4.2) and (4.3), the pseudowavenumber of the finite-difference schemes and the amplification rate of the filters are derived as follows:

$$
\begin{aligned}
& \bar{\kappa}_{i}(\kappa)=\frac{A_{i}(\kappa) D_{i}(\kappa)-B_{i}(\kappa) C_{i}(\kappa)}{A_{i}^{2}(\kappa)+B_{i}^{2}(\kappa)}-j \frac{A_{i}(\kappa) C_{i}(\kappa)+B_{i}(\kappa) D_{i}(\kappa)}{A_{i}^{2}(\kappa)+B_{i}^{2}(\kappa)} \\
& T_{i}(\kappa)=\frac{\tilde{\hat{f}}(k)}{\tilde{f}(k)}=1+\frac{A_{F i}(\kappa) C_{F i}(\kappa)+B_{F i}(\kappa) D_{F i}(\kappa)}{A_{F i}^{2}(\kappa)+B_{F i}^{2}(\kappa)}+j \frac{A_{F i}(\kappa) D_{F i}(\kappa)-B_{F i}(\kappa) C_{F i}(\kappa)}{A_{F i}^{2}(\kappa)+B_{F i}^{2}(\kappa)}
\end{aligned}
$$

which are complex functions of $\kappa \in[0, \pi]$, which varies with nodal position $i$. The component functions in Eqs. (4.2) and (4.4) are given by

$$
\left.\begin{array}{l}
A_{i}(\kappa)=1+\alpha \cos (\kappa)+\beta \cos (2 \kappa) \\
B_{i}(\kappa)=\alpha \sin (\kappa)+\beta \sin (2 \kappa) \\
C_{i}(\kappa)=\sum_{m=0}^{M}\left\{c_{0 m} \cos (m \kappa)+c_{0 m}^{*} \cos [(m+1) \kappa]\right\} \\
D_{i}(\kappa)=\sum_{m=0}^{M}\left\{c_{0 m} \sin (m \kappa)-c_{0 m}^{*} \sin [(m+1) \kappa]\right\}
\end{array}\right\} \text { for } i=0 \text { or } N_{S}
$$




$$
\left.\begin{array}{l}
A_{i}(\kappa)=1+2 \alpha \cos (\kappa)+\beta \cos (2 \kappa) \\
B_{i}(\kappa)=\beta \sin (2 \kappa) \\
\left.C_{i}(\kappa)=\sum_{m=0}^{M}\left\{c_{1 m} \cos [(m-1) \kappa]+c_{1 m}^{*} \cos [(m+2) \kappa]\right\}\right\} \text { for } i=1 \text { or } N_{S}-1, \\
D_{i}(\kappa)=\sum_{m=0}^{M}\left\{c_{1 m} \sin [(m-1) \kappa]-c_{1 m}^{*} \sin [(m+2) \kappa]\right\}
\end{array}\right\} \text { for } 2 \leqslant i \leqslant N_{S}-2 \text { (interior). }
$$

The component functions $\left[A_{F i}(\kappa), B_{F i}(\kappa), C_{F i}(\kappa), D_{F i}(\kappa)\right]$ in Eqs. (4.3) and (4.5) appear the same as those in Eqs. (4.6)-(4.8) with an extra subscript " $F$ " for the coefficients as well as $M_{F}$ instead of $M$, except for the following:

$$
C_{F i}(\kappa)=2 \sum_{m=1}^{3} a_{F m}[\cos (m \kappa)-1] \quad \& \quad D_{F i}(\kappa)=0 \quad \text { for } 2 \leqslant i \leqslant N_{S}-2 \text { (interior). }
$$

It can be found from Eqs. (4.8) and (4.9) that the interior schemes and filters (for $2 \leqslant i \leqslant N_{S}-2$ ) only have real numbers in their pseudo-wavenumber and amplification rate given by Eqs. (4.4) and (4.5). Meanwhile, the SB schemes and filters generate imaginary numbers that might incur significantly different resolution characteristics compared to those of the interior ones. It was indicated in [18] that non-uniform distribution of the pseudo-wavenumber and the amplification rate across
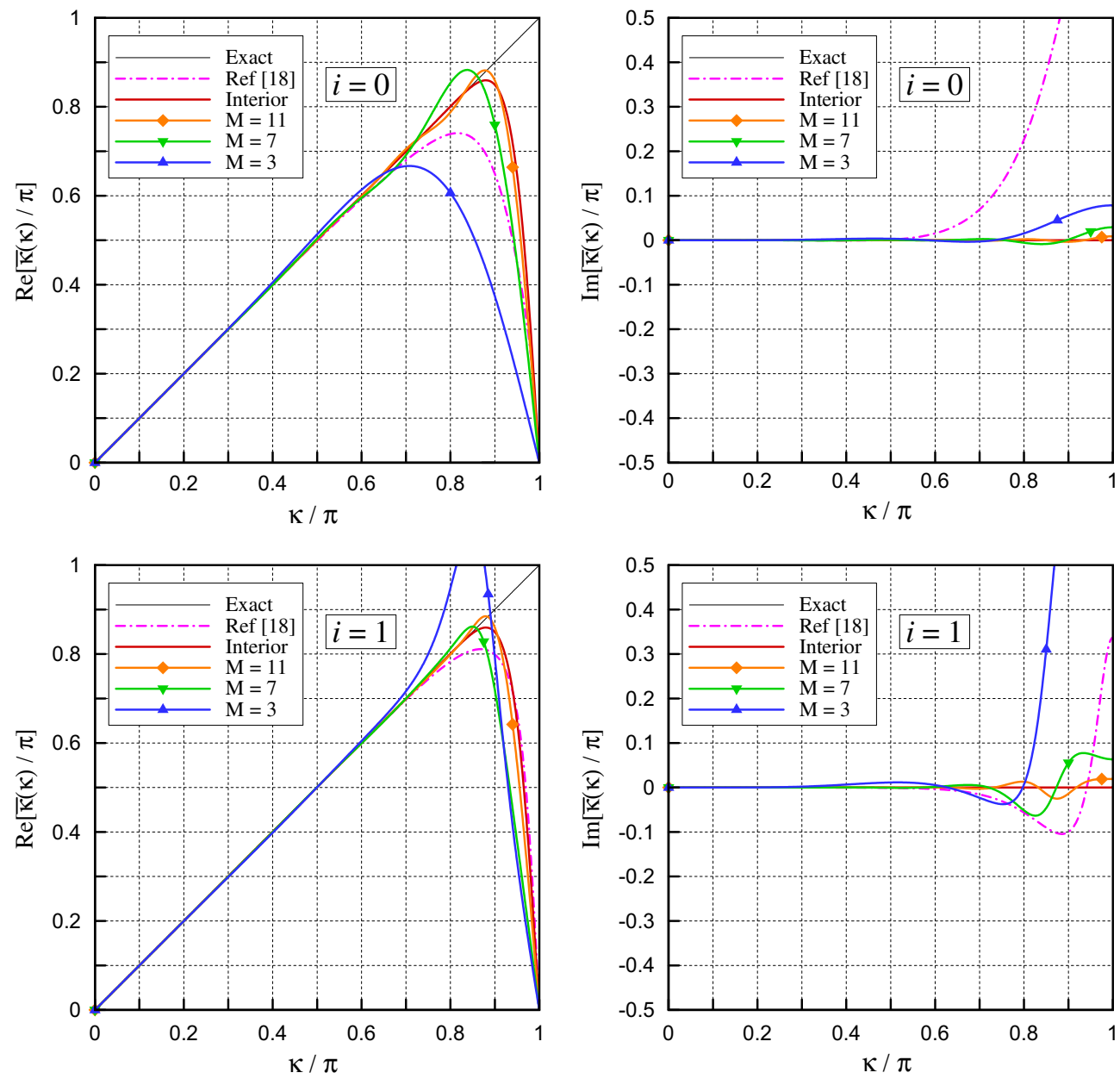

Fig. 4. Pseudo-wavenumber profiles of proposed SB compact schemes in different stencil sizes compared with interior scheme and the earlier work by Kim and Sandberg [18]: real (left) and imaginary (right) parts. 

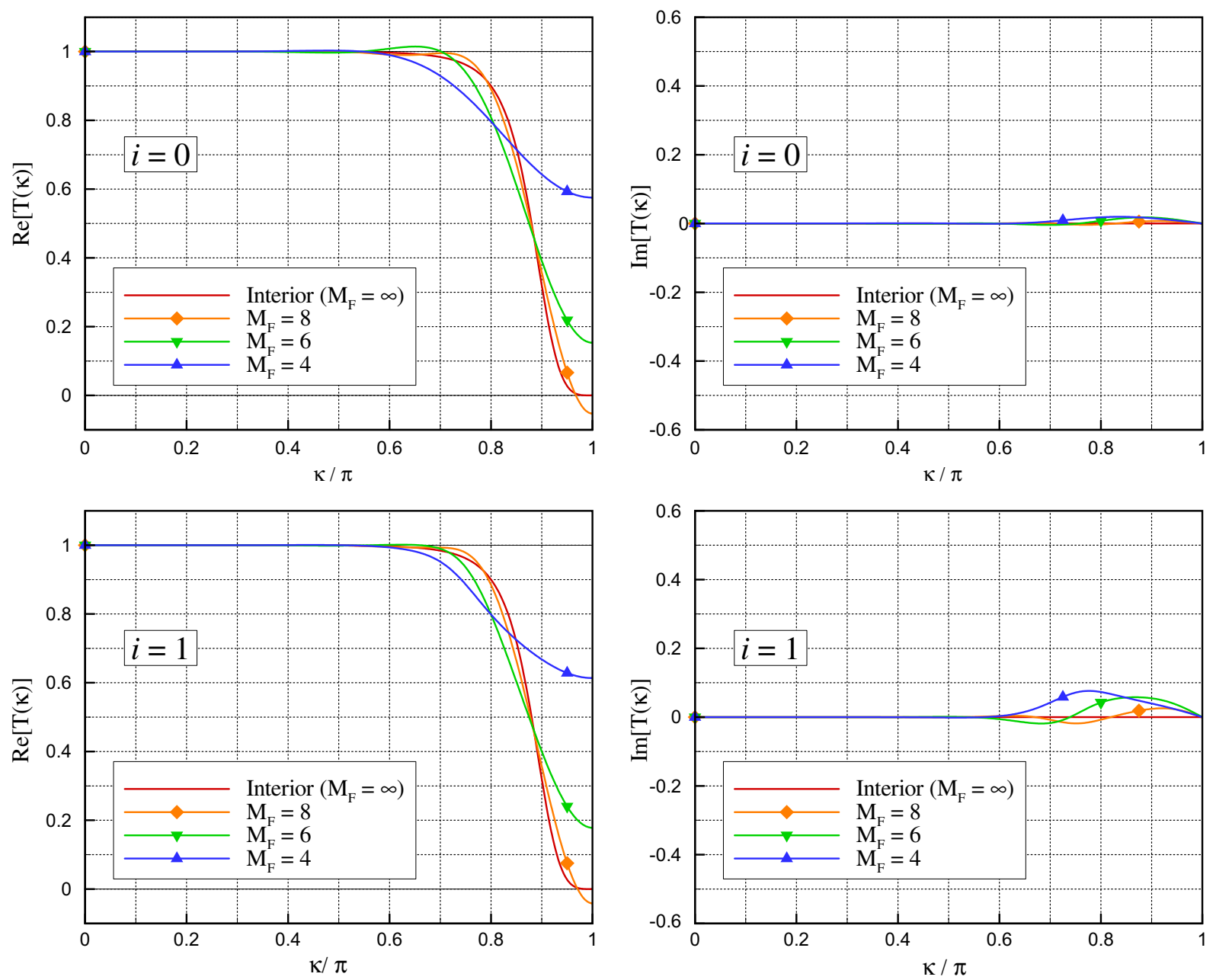

Fig. 5. Amplification rate profiles of proposed SB compact filters in different stencil sizes compared with interior filter: real (left) and imaginary (right) parts.

subdomain boundaries might be the main source of artifacts arising in parallel calculations. The present work aims to substantially reduce the non-uniformity in resolution characteristics across subdomain boundaries. The resolution non-uniformity of the proposed SB schemes and filters with reference to the interior ones may be quantified as follows:

$$
\begin{aligned}
\Phi & =\sqrt{\frac{1}{\pi^{3}} \int_{0}^{\pi}\left[\left|\bar{\kappa}_{0}(\kappa)-\bar{\kappa}_{\text {Interior }}(\kappa)\right|^{2}+\left|\bar{\kappa}_{1}(\kappa)-\bar{\kappa}_{\text {Interior }}(\kappa)\right|^{2}\right] d \kappa}, \\
\Phi_{F} & =\sqrt{\frac{1}{\pi} \int_{0}^{\pi}\left[\left|T_{0}(\kappa)-T_{\text {Interior }}(\kappa)\right|^{2}+\left|T_{1}(\kappa)-T_{\text {Interior }}(\kappa)\right|^{2}\right] d \kappa},
\end{aligned}
$$

which are $L_{2}$-norm based measure of the deviation in resolution characteristics of the SB schemes and filters (denoted by subscripts " 0 " and " 1 ") from the interior ones. It is expected that the resolution non-uniformity decays as the size of the SB stencils ( $M$ and $M_{F}$ ) increases, on which a parameter study is presented below.

Figs. 4 and 5 provide the profiles of the pseudo-wavenumber $\bar{\kappa}_{i}(\kappa)$ and the amplification rate $T_{i}(\kappa)$ from Eqs. (4.4) and (4.5) for different stencil sizes $\left(M\right.$ and $\left.M_{F}\right)$. It is evidenced that the agreement with the interior schemes and filters becomes better as $M$ and $M_{F}$ increases. Fig. 6 shows the history of $\Phi$ and $\Phi_{F}$ converging with increasing $M$ and $M_{F}$ on a logarithmic scale, which indicates that the resolution non-uniformity diminishes exponentially as the size of stencils increases. The calculated value of $\Phi$ from the earlier work by Kim and Sandberg [18] is 0.3146 . Based on the results in Fig. 6, $M=11$ and $M_{F}=8$ are selected as optimal stencil sizes for the present SB compact schemes and filters. The resulting coefficients are provided in Appendix B. The selected stencil sizes maintain the level of $\Phi$ and $\Phi_{F}$ both below 0.03 (3\% criterion). This implies a significant improvement in SB schemes compared to [18], although the SB filters may not be directly compared with those of [18] since the old filters were based on an iterative (predictor-corrector) approach. A much stricter criterion $0.3 \%$ can be achieved at 


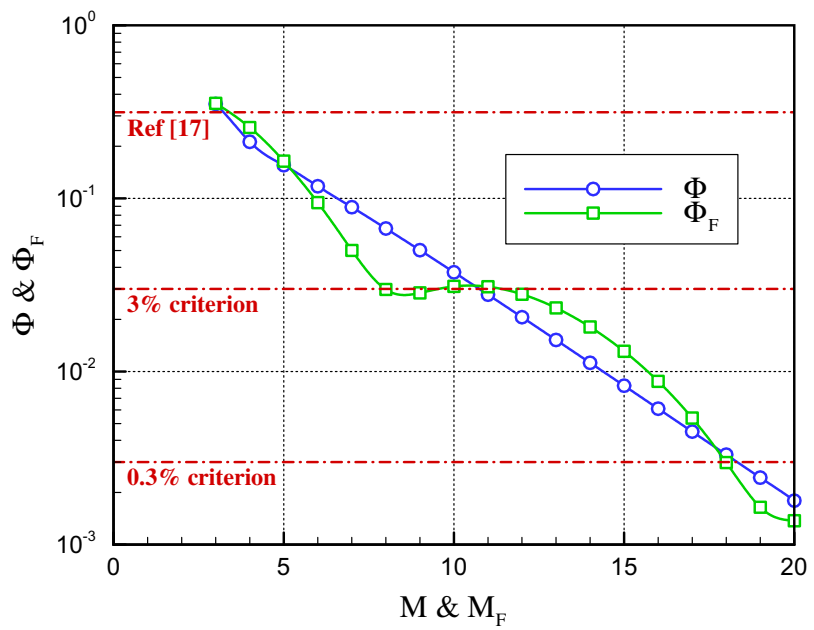

Fig. 6. Convergence history of resolution non-uniformity defined in Eq. (4.10) varying with stencil sizes.
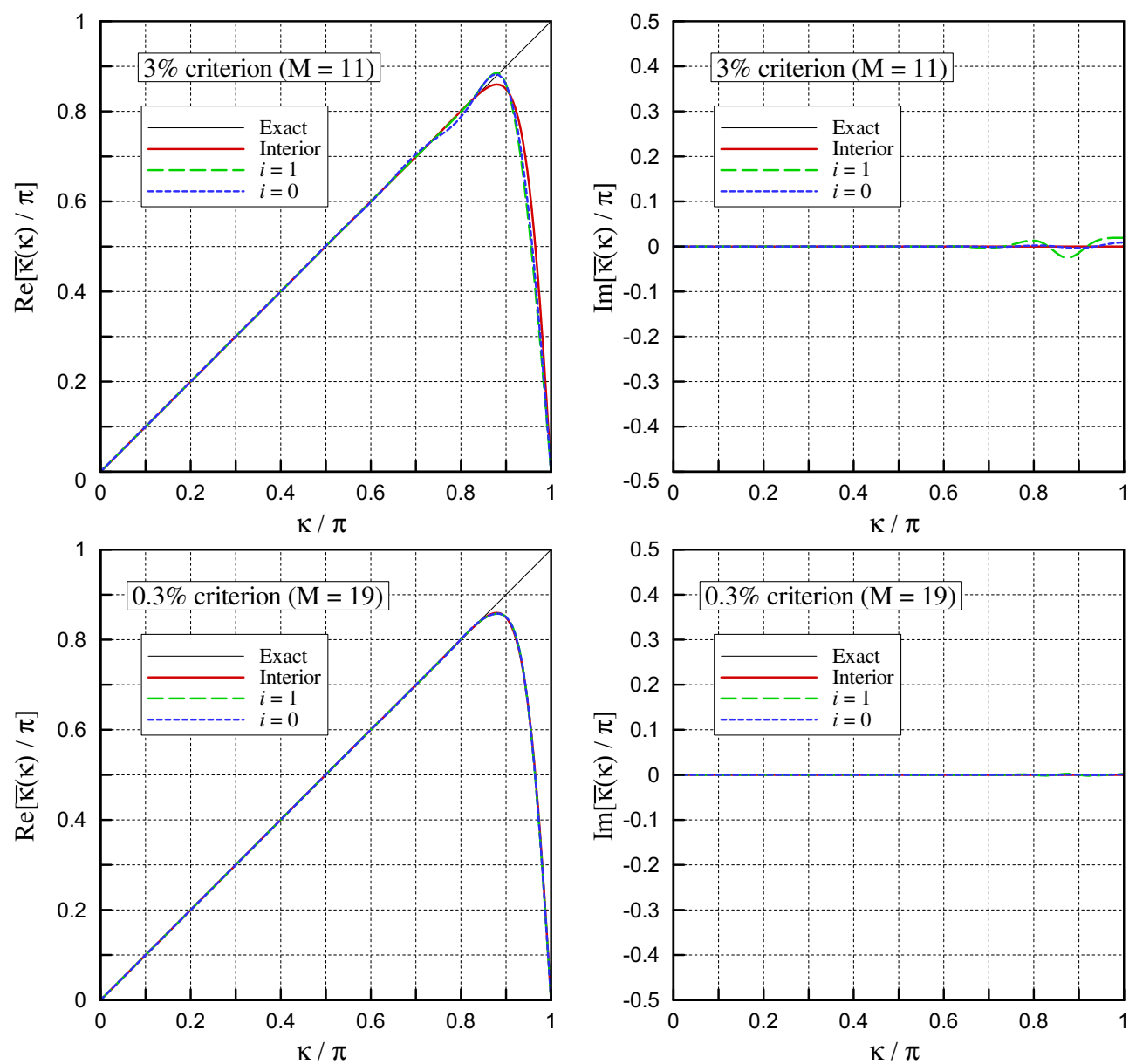

Fig. 7. Pseudo-wavenumber profiles of proposed SB compact schemes for two different criteria of Fourier error defined in Eq. (4.10): $\Phi<0.03$ (top) and $\Phi<0.003$ (bottom)

$M=M_{F}=19$, which may demand a significant drop in parallel efficiency as predicted by Eq. (3.18). In Figs. 7 and 8, the profiles of $\bar{\kappa}_{i}(\kappa)$ and $T_{i}(\kappa)$ are compared between the two different criteria (3\% and $0.3 \%$ ), which showcases that the $3 \%$ criterion 

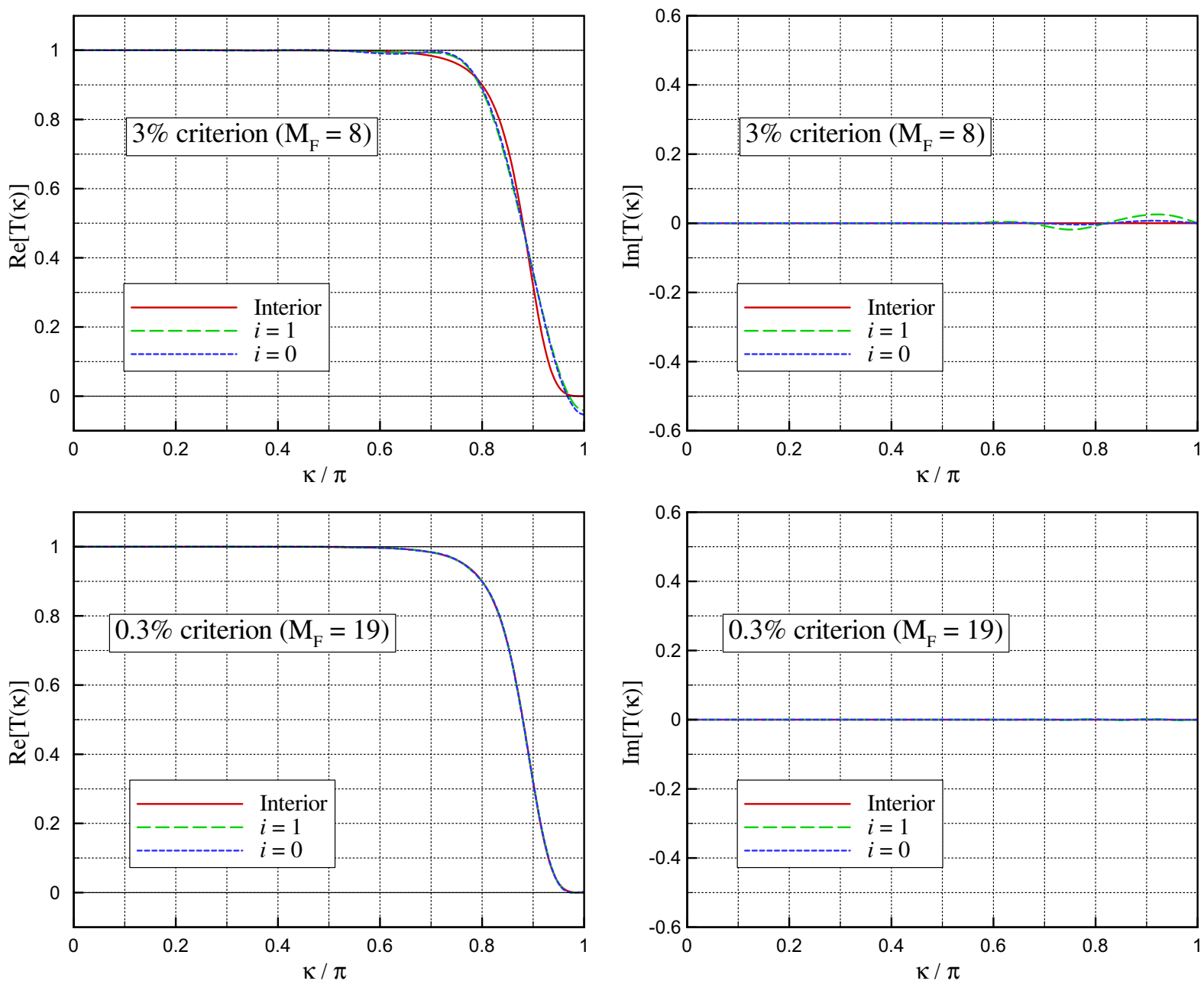

Fig. 8. Amplification rate profiles of proposed SB compact filters for two different criteria of Fourier error defined in Eq. (4.10): $\Phi_{F}<0.03$ (top) and $\Phi_{F}<0.003$ (bottom).

provides reasonably well matched profiles with the interior ones and the $0.3 \%$ criterion leads ultimately to an artifact-free condition.

\subsection{Linear stability analysis}

The stability analysis of the proposed SB compact schemes and filters begins by considering a one-dimensional linear scalar wave equation:

$$
\partial_{t} f+c_{\infty} \partial_{x} f=0
$$

over a subdomain $x \in[0, L]$ where $c_{\infty}$ is the wave convection speed ( $\left.c_{\infty}>0\right)$. Complying with the direction of the wave, a prescribed boundary condition is required in the upstream domain $(x<0)$. Current setup for the stability analysis is that the proposed SB compact schemes and filters are used at the left boundary $(x=0)$, and the upstream boundary condition is provided via halo terms from a neighboring subdomain on the left. Ordinary boundary schemes and filters (with $\varepsilon_{F}=0$ in Appendix A) given by Eqs. (2.1) and (2.3) are used at the right boundary $(x=L)$ through which the prescribed wave leaves the domain. This problem setup corresponds effectively to Eq. (3.8). The upstream boundary condition here can be set to zero, $f_{B C}(x<0, t)=0$ without loss of generality [2]. With this boundary condition in place, Eq. (3.8) may reduce to:

$$
\overline{\mathbf{f}}^{\prime}=\frac{1}{\Delta x} \mathbf{R}_{B}^{-1} \mathbf{S}_{B}(\mathbf{f}+\widehat{\Delta} \mathbf{f})=\frac{1}{\Delta x} \mathbf{R}_{B}^{-1} \mathbf{S}_{B}\left(\mathbf{I}+\mathbf{R}_{F B}^{-1} \mathbf{S}_{F B}\right) \mathbf{f}
$$

which represents a combined differencing-filtering system. $\mathbf{I}$ is an identity matrix. The subdomain is discretized into $N_{S}$ equal intervals with $\Delta x=L / N_{S}$ and the discretization index runs from $i=0$ to $i=N_{S}$. 


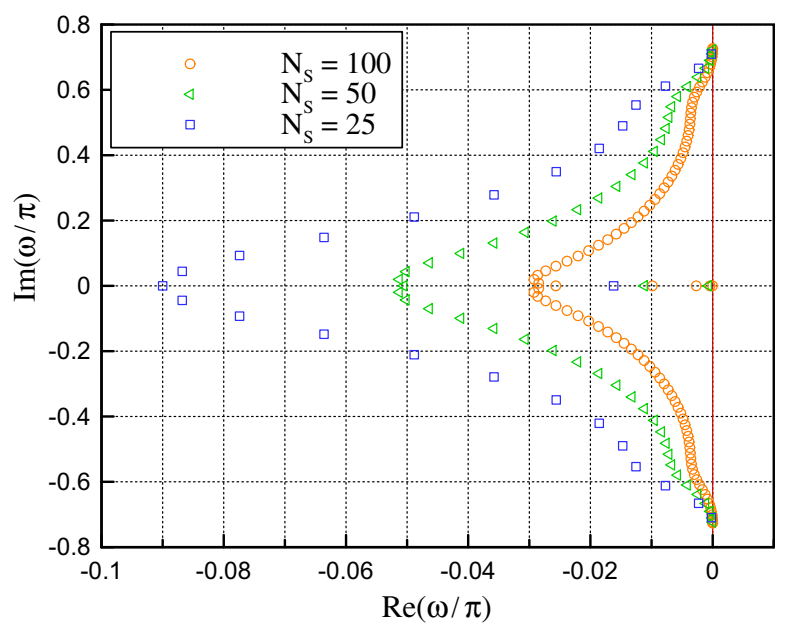

Fig. 9. Distribution of eigenvalues of Eq. (4.14) in different numbers of grid cells $\left(N_{S}\right)$ used.

Relating Eqs. (4.11) and (4.12) leads to the following equation:

$$
\frac{d \mathbf{f}}{d t}=-\frac{c_{\infty}}{\Delta x} \mathbf{R}_{B}^{-1} \mathbf{S}_{B}\left(\mathbf{I}+\mathbf{R}_{F B}^{-1} \mathbf{S}_{F B}\right) \mathbf{f} .
$$

Since Eq. (4.13) is a system of ordinary differential equations with respect to time with constant coefficients the solution consists of normal modes $\mathbf{f}=\mathbf{f}_{0} e^{w t}$ with a constant $w$ representing the rates of decay or amplification of the modes. Imposing the normal modes into Eq. (4.13) reduces to an eigenvalue problem:

$$
-\mathbf{R}_{B}^{-1} \mathbf{S}_{B}\left(\mathbf{I}+\mathbf{R}_{F B}^{-1} \mathbf{S}_{F B}\right) \mathbf{f}_{0}=\omega \mathbf{f}_{0}
$$

where $\omega=w \Delta x / c_{\infty}$ is the dimensionless eigenvalue (complex number) and $\mathbf{f}_{0}$ becomes the corresponding eigenvector. The real parts of the eigenvalues are required to be equal to or less than zero to ensure numerical stability of the system, i.e. $\left|e^{w} t\right| \leqslant 1$. Fig. 9 shows the distribution of eigenvalues in the complex plane where all the eigenvalues are located in the left half plane proving linear stability of the present differencing-filtering system.

\section{Application to benchmark problems}

In this section the performance of the proposed SB compact finite-difference schemes and filters is demonstrated through their application to multi-domain parallel calculations for benchmark test cases. As recommended in Section 4.1, $M=11$ and $M_{F}=8$ are consistently used in this section. The benchmark tests are based on one-dimensional linear scalar wave equation and two-dimensional nonlinear compressible Euler equations. In all cases, a standard fourth-order Runge-Kutta method is used for temporal marching of the numerical solutions. The compact filters are implemented for conservative variables at the last stage in each time step of the Runge-Kutta routine. The accuracy and efficiency of the present calculations are quantified by comparing with analytic solutions and data from single-domain serial calculations.

\subsection{Linear scalar wave convection}

The one-dimensional linear wave convection in Eq. (4.11) is calculated as a canonical benchmark test case. The initial wave profile and the upstream boundary condition are given by

$$
\begin{aligned}
& f(x, t=0)=\left[2+\cos \left(k_{1} x / L\right)\right] \exp \left[-(\ln 2)\left(k_{2} x / L\right)^{2}\right] \text { for } x \in[-0.5 L, 1.5 L], \\
& f(x<-0.5 L, t)=f(-0.5 L, 0) \quad \& \quad \partial_{x} f(x<-0.5 L, t)=0,
\end{aligned}
$$

where $L$ is an arbitrary characteristic length; and the constants are set to $k_{1}=17 k_{2}$ and $k_{2}=10$. The calculation runs until the center of the wave pulse travels from $x / L=0$ to 1 over a time period of $c_{\infty} t / L=1$. The domain is split into four subdomains: $x \in[-0.5 L, 0],[\Delta x, 0.5 L],[0.5 L+\Delta x, L]$ and $[L+\Delta x, 1.5 L]$, which have $N / 4+1, N / 4, N / 4$ and $N / 4$ number of grid points $(N+1$ in total), respectively. The grid points are equally spaced, i.e. $\Delta x=2 L / N$. The proposed SB compact schemes and filters are implemented across each subdomain boundary. The time step size is set to $c_{\infty} \Delta t / L=N^{-1}$ corresponding to the CFL number of 0.5 at which the contribution of the temporal marching to the overall solution errors is insignificant.

Fig. 10 shows the result of calculation comparing the present method with others. The present parallel solution agrees very well with the serial solution as well as with that from the previous approach of [18]. It is also noticeable that the family 

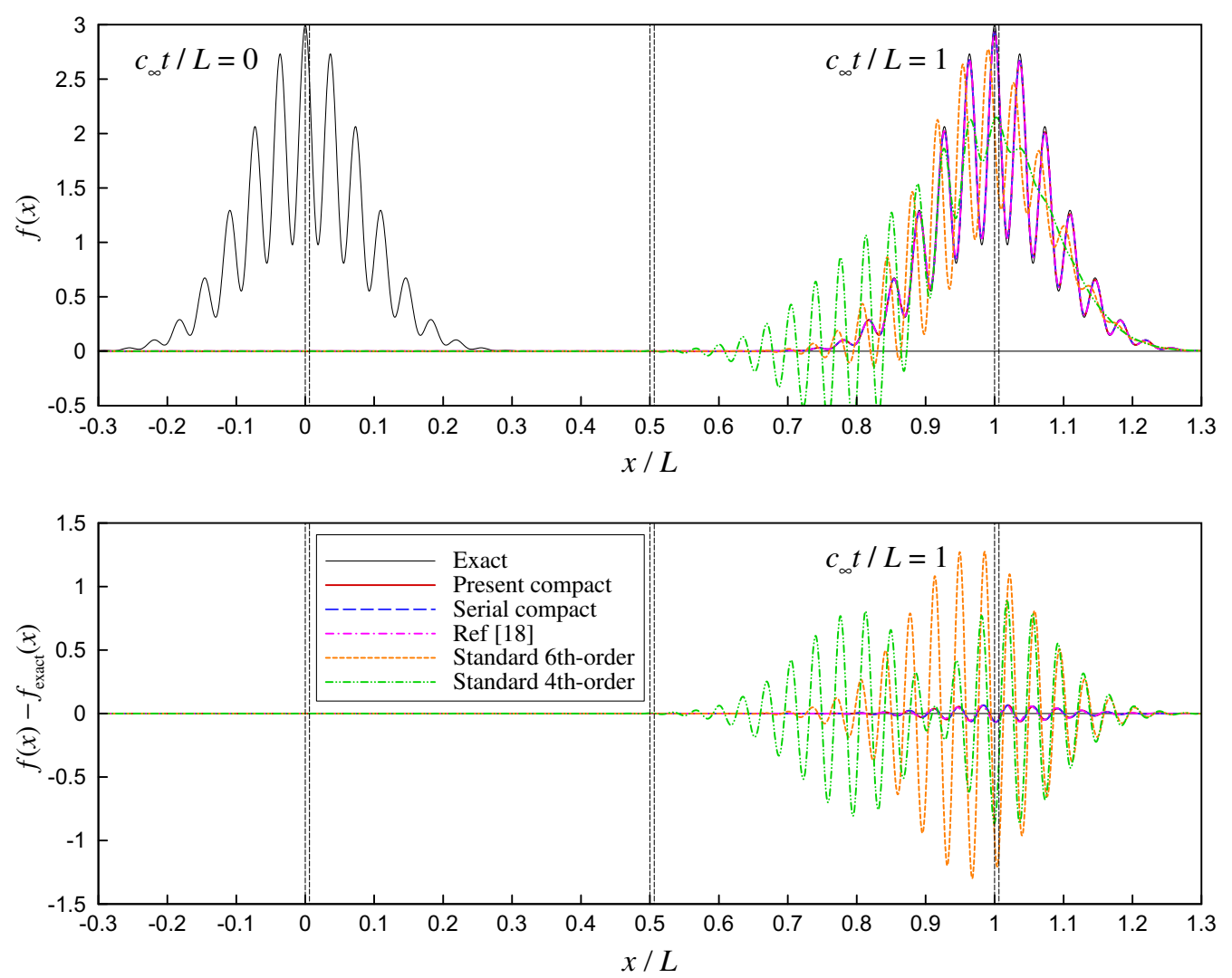

Fig. 10. Parallel calculations of one-dimensional linear wave convection: calculated wave profiles (top) and numerical errors from exact solution (bottom). Four subdomains with $N_{S}=(80,79,79,79)$ and $N=320$ in total. Dashed lines denote the location of subdomain boundaries.
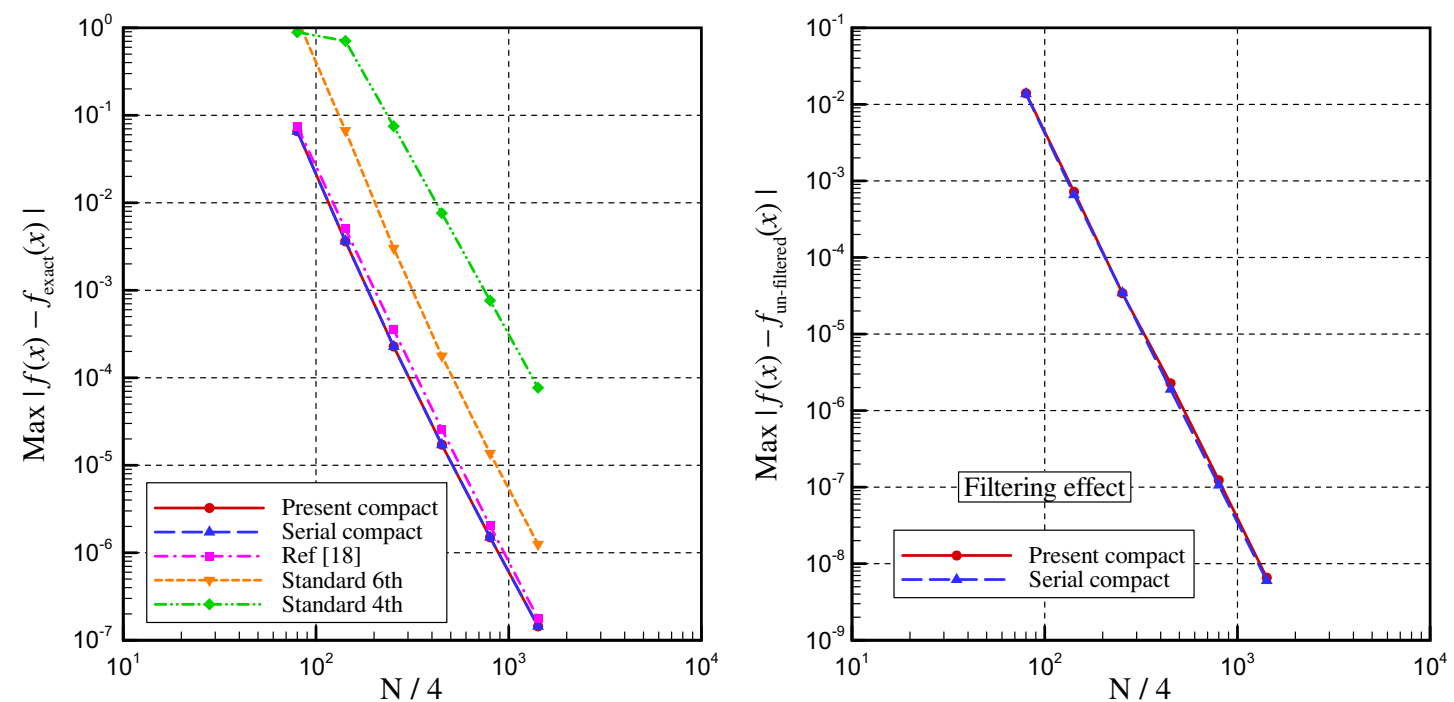

Fig. 11. Convergence history of numerical error (left) and filter's contribution (right) on $L_{\infty}$-norm basis varying with the number of grid points used in the calculation of one-dimensional linear wave convection. Quantities are measured at $c_{\infty} t / L=1$.

of compact schemes outperforms the standard explicit schemes on a reasonably small number of grid points (the standard explicit schemes were used with the present compact filters in order to keep consistency in comparing the major effect of finite-difference schemes). Fig. 11 plots the convergence history of numerical error and filter's contribution 


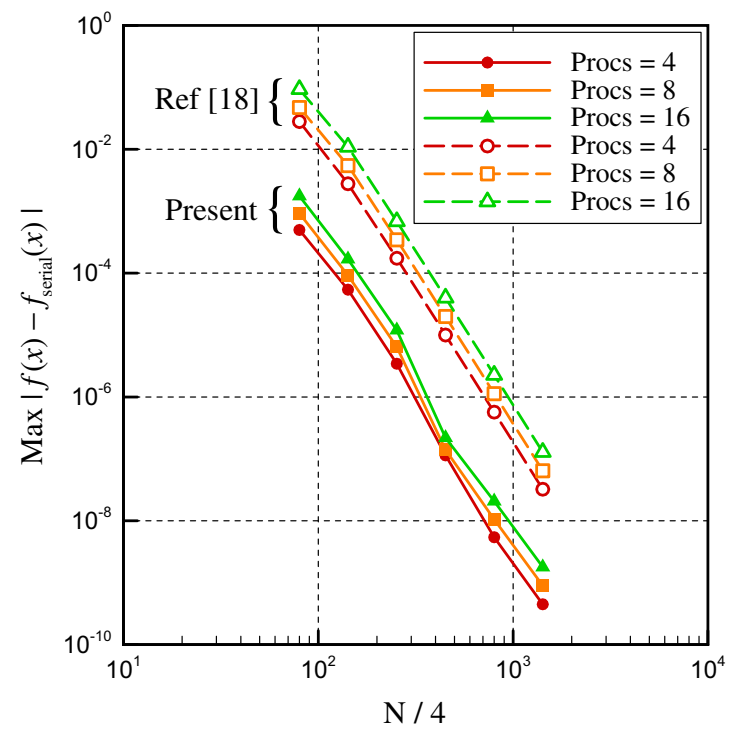

Fig. 12. Convergence history of difference between parallel and the serial solutions on $L_{\infty}$-norm basis varying with the number of grid points used for different number of processor-cores in the calculation of one-dimensional linear wave convection. Quantities are measured at $c_{\infty} t / L=1$.

$\left(\operatorname{Max}\left|f(x)-f_{\text {exact }}(x)\right|\right.$ and $\left.\operatorname{Max}\left|f(x)-f_{\text {un-filtered }}(x)\right|\right)$ defined on $L_{\infty}$-norm basis varying with the number of grid points used. It confirms the expected fourth-order accuracy of the present method along with others. However, the standard sixth-order scheme yields a fifth-order convergence rate. This is due to the fifth-order accuracy of the filters as revealed in an additional plot of Fig. 11. The sixth-order interior filters blended with the fourth-order boundary filters, i.e. Eqs. (2.3) and (2.4) resulted in an fifth-order accuracy overall.

It is shown in Fig. 11 that the present parallel calculation reproduces almost identical convergence history to that of the serial one whereas the previous approach by Kim and Sandberg [18] yields a noticeable difference. The fidelity of the present parallel calculations can be judged by the level of agreement with the corresponding serial calculations. Fig. 12 plots the difference between parallel and the serial solutions defined on $L_{\infty}$-norm basis $\left(\operatorname{Max}\left|f(x)-f_{\text {serial }}(x)\right|\right)$ varying with the number of grid points used. The calculations are also performed for larger numbers of processor-cores ( 8 and 16$)$. It is found that the present parallel computing approach improves the level of agreement with the serial solutions by a factor of 100 compared to the earlier approach by Kim and Sandberg [18]. It is also observed (by comparing Figs. 11 and 12) that the level of artifacts in the present parallel solutions (deviation from the serial solutions) is two orders of magnitude smaller than the genuine numerical error (deviation from the exact solutions) regardless of grid resolution for up to 16 processor-cores used across the one-dimensional domain.

\subsection{Isentropic vortex convection}

The second benchmark test is the convection of two-dimensional isentropic vorticity waves in a subsonic free stream. An initial isentropic vorticity wave packet located in the upstream part of the computational domain moves downstream and passes through subdomain boundaries in a parallel computing setup. The simulation is based on full nonlinear compressible Euler equations in a conservative form in generalized coordinates, which can be found in [18]. It was indicated in [18] that a vortex crossing subdomain boundaries might generate spurious reflections up to a level that interferes the genuine hydrodynamic/acoustic field. This might become a critical issue in DNS of a subsonic jet where quadrupole noise due to unsteady vortices dominates. The current benchmark test aims to demonstrate a significant reduction of such acoustic reflections by using the proposed method compared to the latest results in [18]. The isentropic vortex is described by the following equations over a rectangular domain of $x \in[-0.5 L, 0.5 L]$ and $y \in[-0.5 L, 0.5 L]$ :

$$
\left.\begin{array}{l}
\rho(x, y, t)=\rho_{\infty}\left[1-\frac{\gamma-1}{2} \psi^{2}\left(x^{*}, y\right)\right]^{1 /(\gamma-1)} \\
u(x, y, t)=u_{\infty}+c_{\infty}\left(k_{1} y / L\right) \psi\left(x^{*}, y\right) \\
v(x, y, t)=-c_{\infty}\left(k_{1} x^{*} / L\right) \psi\left(x^{*}, y\right) \\
p(x, y, t)=p_{\infty}\left(\rho / \rho_{\infty}\right)^{\gamma}
\end{array}\right\} \quad \text { with } \quad\left\{\begin{array}{l}
\psi(x, y)=k_{2} \exp \left[-\frac{1}{2} k_{1}^{2}\left(x^{2}+y^{2}\right) / L^{2}\right] \\
x^{*}=x-x_{o}-u_{\infty} t .
\end{array}\right.
$$

The size and the strength of the vortex are controlled by $k_{1}$ and $k_{2}$, respectively. Various sizes and strengths are tested. Initial location of the vortex core is $(x, y)=\left(x_{0}, 0\right)$ where $x_{o}=-0.125 \mathrm{~L}$ is used. The free stream velocity $u_{\infty}=M_{\infty} c_{\infty}$ is given by the Mach number $M_{\infty}=0.5$, where $c_{\infty}=\left(\gamma p_{\infty} / \rho_{\infty}\right)^{1 / 2}$ is the ambient speed of sound. The calculations are carried out on three dif- 
ferent (one uniform and two non-uniform) grids with a fixed number of cells $(N \times N)=(200 \times 200)$. The grids are generated by the following formula used by Gaitonde and Visbal [7]:

$$
\left.\begin{array}{l}
x_{i, j} / L=-\frac{1}{2}+\frac{1}{N}[i+\varepsilon \sin (4 \pi j / N)] \\
y_{i, j} / L=-\frac{1}{2}+\frac{1}{N}[j+\varepsilon \sin (4 \pi i / N)]
\end{array}\right\} \text { for } 0 \leqslant i \leqslant N \text { and } 0 \leqslant j \leqslant N
$$

which gives curvilinear grids deformed from a uniform grid by controlling the constant $\varepsilon$. Setting $\varepsilon=0$ restores the uniform grid. Two different grids with $\varepsilon=0$ and 3 are tested. Non-reflecting boundary conditions [20] are implemented at the outer boundaries with the time derivatives of all the incoming waves set to zero.

The domain is split into two (left and right) and then four (top and bottom in addition) subdomains to see the effect of multi-directional decomposition. The calculations run until $u_{\infty} t / L=0.25$ by which the vortex has travelled a quarter of the domain length after passing through the subdomain boundaries in the middle. The time step size is fixed at $u_{\infty} \Delta t / L=(25 /$ $6) \times 10^{-4}$ which corresponds to the CFL number of 0.5 in the case of uniform grid. All the results are compared with reference data from single-domain serial calculation. In order to examine the accuracy of calculations properly, the following quantity is introduced:

$$
P_{S}=\frac{\left|p-p_{\text {serial }}\right|}{p_{\infty}-p_{\text {min }}}=\frac{\left|p-p_{\text {serial }}\right|}{p_{\infty}\left[1-\left(1-\frac{\gamma-1}{2} k_{2}^{2}\right)^{\gamma /(\gamma-1)}\right]}
$$

which gives the difference of pressure between the parallel and the serial solutions normalized by the pressure drop at the vortex core where the pressure is minimum. This normalization provides self-similarity in plotting the pressure field, which allows fair comparison between cases with different vortex strengths $\left(k_{2}\right)$. The distribution of $P_{S}$ taken at the last moment $u_{\infty} t / L=0.25$ is plotted in three different logarithmic scales in Fig. 13 (for two subdomains) and 14 (for four subdomains) comparing the results of using the present method and the previous approach of [18]. The vortex diameter (based on the definition given in [18]) is moderately resolved by 17 cells and the core pressure drop is $3.974 \%$ of the ambient pressure $\left(p_{\infty}\right.$ $\left.-p_{\min }=0.03974 p_{\infty}\right)$. It is shown in Fig. 13 and 14 that the present approach substantially reduces the level of parallelization artifacts from subdomain boundaries by at least an order of magnitude compared to the previous approach in [18]. It can also be seen in the figures that the artifact acoustic reflection in the present approach is between $10^{-5}$ and $10^{-6}$ times the hydrodynamic fluctuation (the pressure drop at the vortex core). In fact, Fig. 14 showcases a particularly severe test case where the vortex constantly goes through the lateral subdomain boundary, in which the previous approach [18] generates more reflections than it does in Fig. 13 whereas the present approach makes no noticeable difference.

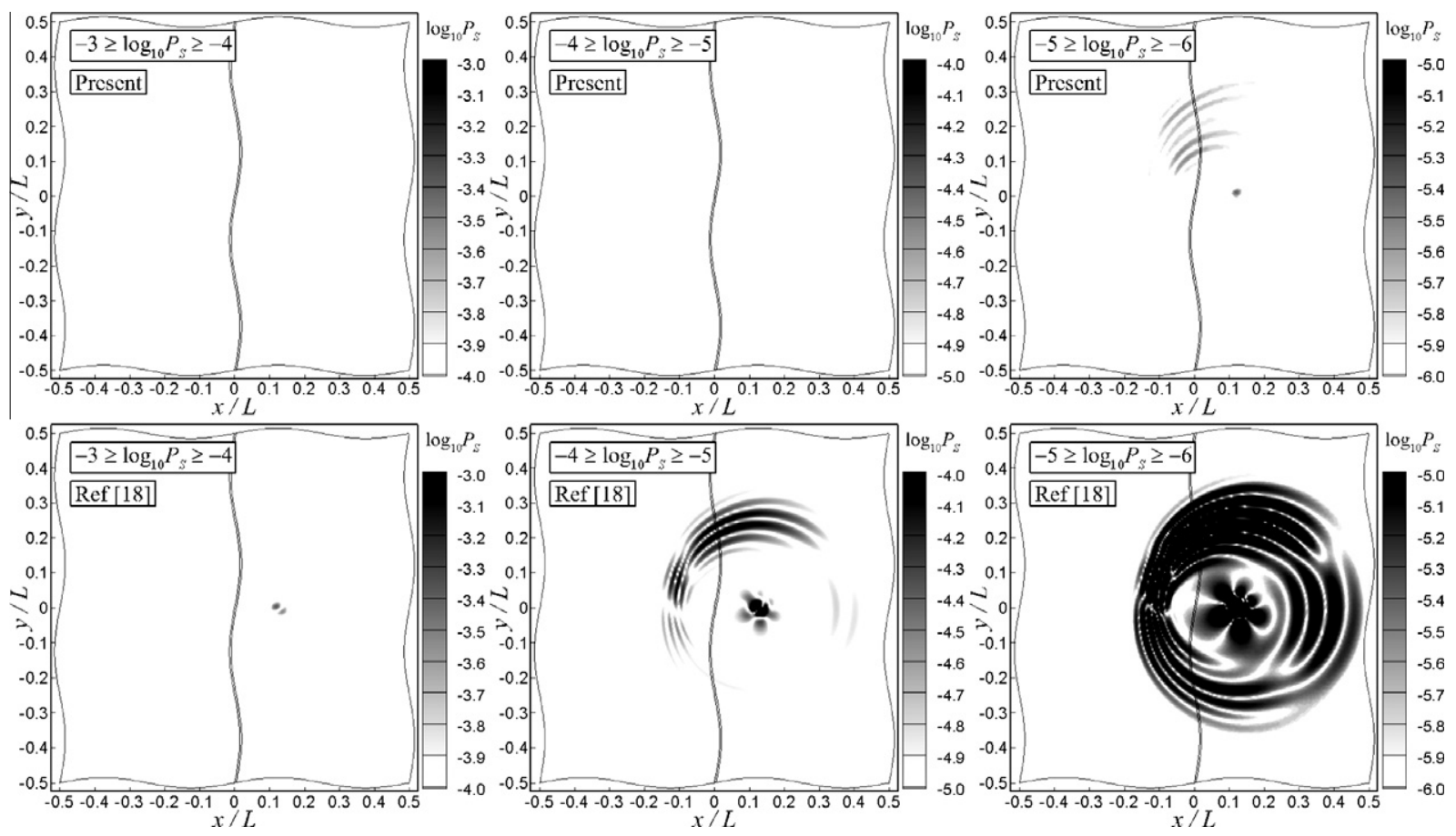

Fig. 13. Distribution of $P_{S}$ defined in Eq. (5.5) plotted at $u_{\infty} t / L=0.25$ from the calculation of isentropic vortex convection with $\left(k_{1}, k_{2}\right)=(50,0.24)$ in Eq. $(5.3)$ on a non-uniform grid with $\varepsilon=3$ in Eq. (5.4). Contour levels in three different ranges in logarithmic scales. Cases with two subdomains by using the present approach (top) and Ref. [18] (bottom). 

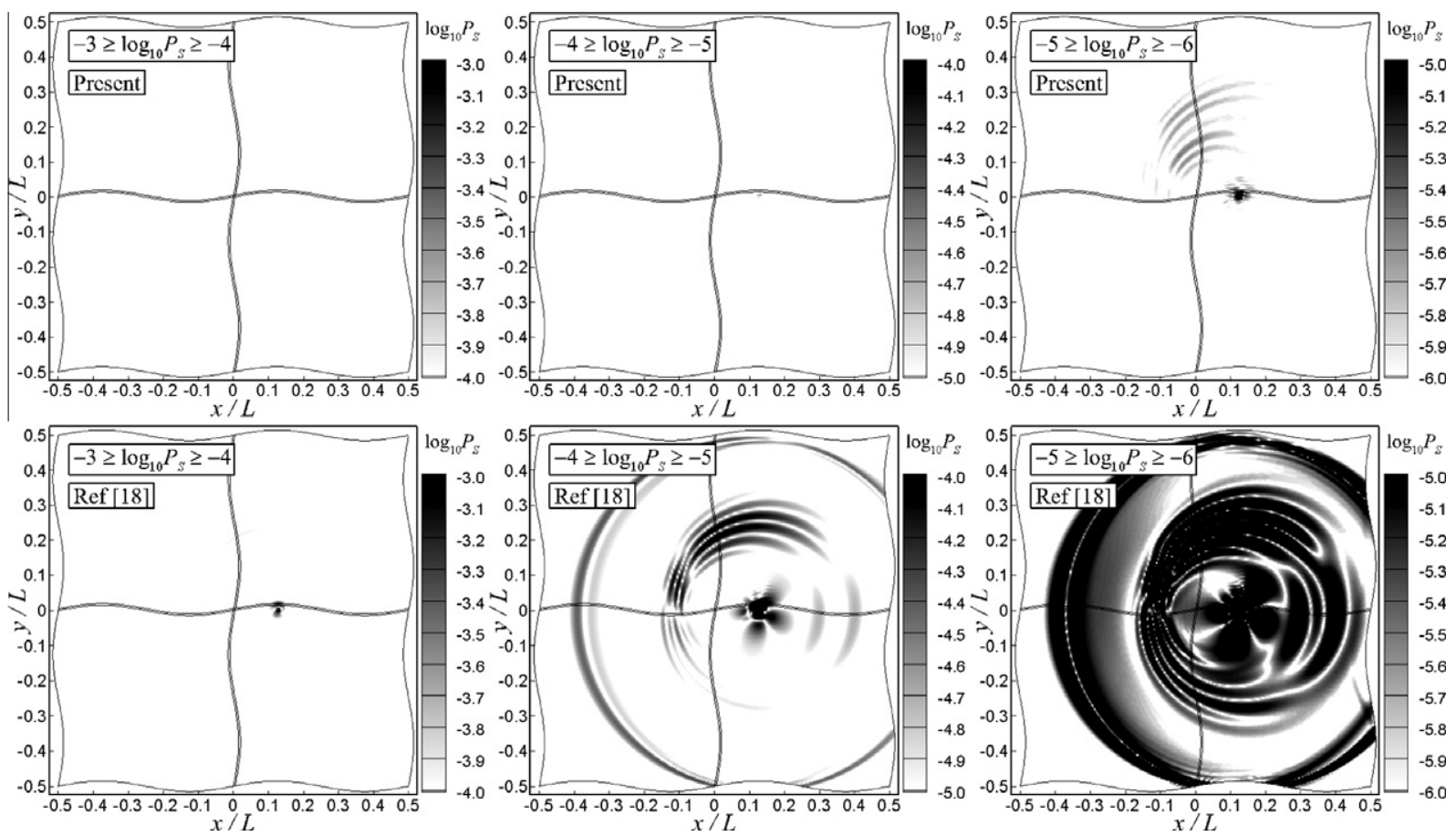

Fig. 14. Distribution of $P_{S}$ defined in Eq. (5.5) plotted at $u_{\infty} t / L=0.25$ from the calculation of isentropic vortex convection with $\left(k_{1}, k_{2}\right)=(50,0.24)$ in Eq. (5.3) on a non-uniform grid with $\varepsilon=3$ in Eq. (5.4). Contour levels in three different ranges in logarithmic scales. Cases with four subdomains by using the present approach (top) and Ref. [18] (bottom).

A more quantitative investigation on $P_{S}$ defined by Eq. (5.5) is carried out in Figs. 15 and 16 where different values of the vortex parameters $k_{1}$ and $k_{2}$ in Eq. (5.3) and the mesh deformation level $\varepsilon$ in Eq. (5.4) are used on two different domains split by two and four subdomains (denoted by S2 and S4). The constant $k_{1}=30$ to 70 corresponds to the diameter of the vortex covered by 28.7 cells (well-resolved) to 12.3 (under-resolved). The other constant $k_{2}=0.08$ to 0.4 changes the pressure drop at the vortex core (hydrodynamic fluctuation) from $p_{\infty}-p_{\min }=0.004473 p_{\infty}$ (linear) to $0.1076 p_{\infty}$ (nonlinear). Fig. 15 plots the largest values of $P_{S}$ obtained at the end of each calculation, which shows the level of artifacts (deviation from the serial solutions) arising mainly in the vicinity of the vortex core - hydrodynamic artifact. The present hydrodynamic artifact falls below $10^{-5}$ (of the core pressure drop) in the well-resolved range while it is maintained at $10^{-4}$ or lower in the under-resolved range with the non-uniform grid $(\varepsilon=3)$ and the severe condition (S4). The results are consistent in both linear and nonlinear cases; and, also in both uniform and non-uniform grids.

As mentioned earlier, a critical issue in the current BAA (boundary approximation approach) is the acoustic artifact that emerges and propagates in all directions at the speed of sound as displayed in Figs. 13 and 14. In order to focus on the acoustic artifact, it is necessary to apply a window function $W_{\text {Acoustic }}$ to $P_{S}$ and phase out the hydrodynamic contribution that is usually larger than the acoustic counterpart, as follows:

$$
P_{S} W_{\text {Acoustic }}=P_{S}\left[1-\psi\left(x^{*}, y\right) / k_{2}\right]=P_{S}\left\{1-\exp \left[-\frac{1}{2} k_{1}^{2}\left(x^{* 2}+y^{2}\right) / L^{2}\right]\right\}
$$

where $x^{*}=x-x_{0}-u_{\infty} t$ is the location of the travelling vortex core as described in Eq. (5.3). Fig. 16 plots the largest values of $P_{S} W_{\text {Acoustic }}$ obtained at the end of each calculation. It is shown that the acoustic artifact in the present approach drops further down below $10^{-6}$ in the well-resolved range and stays in the region of $10^{-5}$ even when the grid resolution is insufficient to properly reproduce the vortex profile. The results are consistent in both linear and nonlinear cases; and, in both uniform and non-uniform grids as well. Compared to the previous approach [18] overall, the present approach provides significantly lower level of artifacts by a factor of 10 to 100 from both hydrodynamic and acoustic perspectives.

Aggregate CPU time elapsed per time step per grid point in an Intel Xeon W3520 quad-core CPU at $2.67 \mathrm{GHz}$ (with the computer code compiled by PGI Visual Fortran 2010) is measured and listed in Table 6 for each domain topology. The table reveals that the present approach offers higher parallel computing efficiency than that of the previous approach [18]. This is mainly due to the fact that the present compact filters do not require an iterative routine, i.e. predictor-corrector steps (twice the effort) which the previous approach [18] employed. The actual efficiencies are compared with the analytical prediction derived in Eq. (3.18) as well. Although the analytical prediction based on an assumption that every single arithmetic operation takes the same CPU time does not entirely correspond to the Euler solver used in this simulation, it provides reasonably close estimation to the actual results $\left(M=11\right.$ and $M_{F}=8$ for the present approach, and $M=M_{F}=3$ for [18], with $N_{S}=100$; and the filters are implemented at the last stage of the Runge-Kutta routine). 

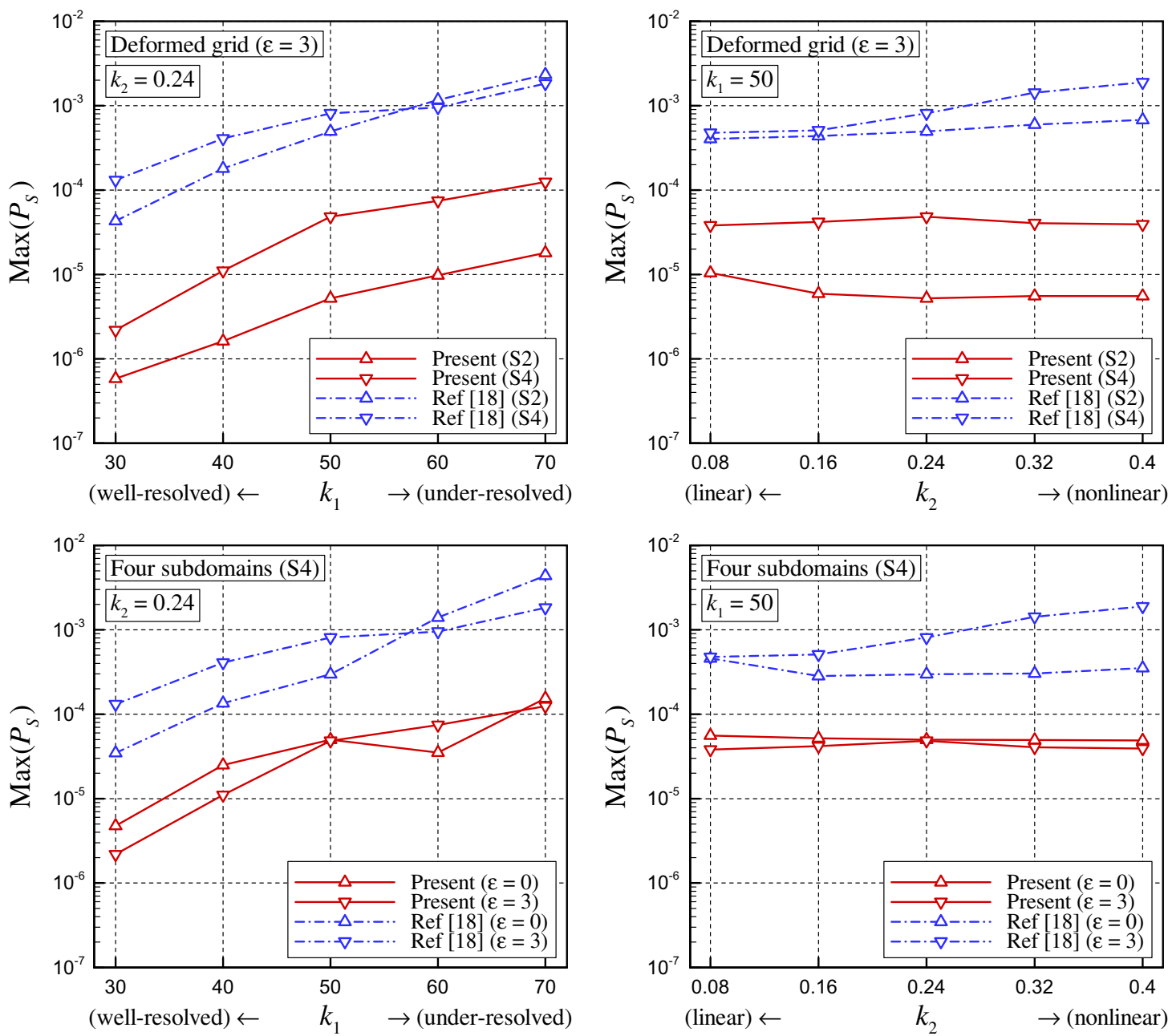

Fig. 15. Plots of largest values of $P_{S}$ from Eq. (5.5) measured at $u_{\infty} t / L=0.25$ from the calculation of isentropic vortex convection with various parameters. S2 and S4 denote cases for two (split in streamwise direction) and four subdomains (split in lateral direction in addition to former).

\subsection{Sound generated by a pair of co-rotating vortices}

The third benchmark test case in the present study is direct calculation of quadrupole sound generated by a pair of corotating vortices in a stationary medium. Co-rotating vortices have been studied as one of the major noise source mechanisms in free shear flows [21], which demand very precise simulations since quadrupole sound is normally the weakest one in subsonic media compared to monopole and dipole ones. This benchmark test will gauge the fidelity of using the proposed parallel computing approach for highly sophisticated aeroacoustics simulations. In addition, this benchmark test is designed to investigate the effect of repetitive collisions between vortices and subdomain boundaries by locating the corotating vortex pair on a junction of multiple subdomains. A question may be asked as to whether a more pronounced level of acoustic artifacts than is estimated in Section 5.2 might develop in the presence of repetitive collisions between vortices and subdomain boundaries that normally take place in massively parallel simulations. The answer to this question will be revealed at the end of the test.

The current test is based on a modified Scully vortex model that provides an initial condition as follows:

$$
\left.\begin{array}{l}
\left.\rho\right|_{x, y, t=0}=\rho_{\infty}\left[1-\frac{\gamma-1}{2}\left(u^{2}+v^{2}\right)\right]^{1 /(\gamma-1)} \\
\left.(u, v)\right|_{x, y, t=0}=\sum_{m=0}^{1} \frac{(1+\delta) \Gamma_{0}}{2 \pi\left(r_{m}^{2}+r_{c}^{2}\right)}\left(y,-x_{m}\right) \\
\left.p\right|_{x, y, t=0}=p_{\infty}\left(\rho / \rho_{\infty}\right)^{\gamma}
\end{array}\right\} \text { with } r_{m}^{2}=x_{m}^{2}+y^{2} \text { and } x_{m}=x+(-1)^{m} L
$$

where $\Gamma_{o}$ is the desired circulation of the velocity field; $\delta$ is a correction factor (to be explained below); and the characteristic length $L$ is the half of the distance between the two vortex cores, i.e. the radius of the circular trajectory of the vortices. The 

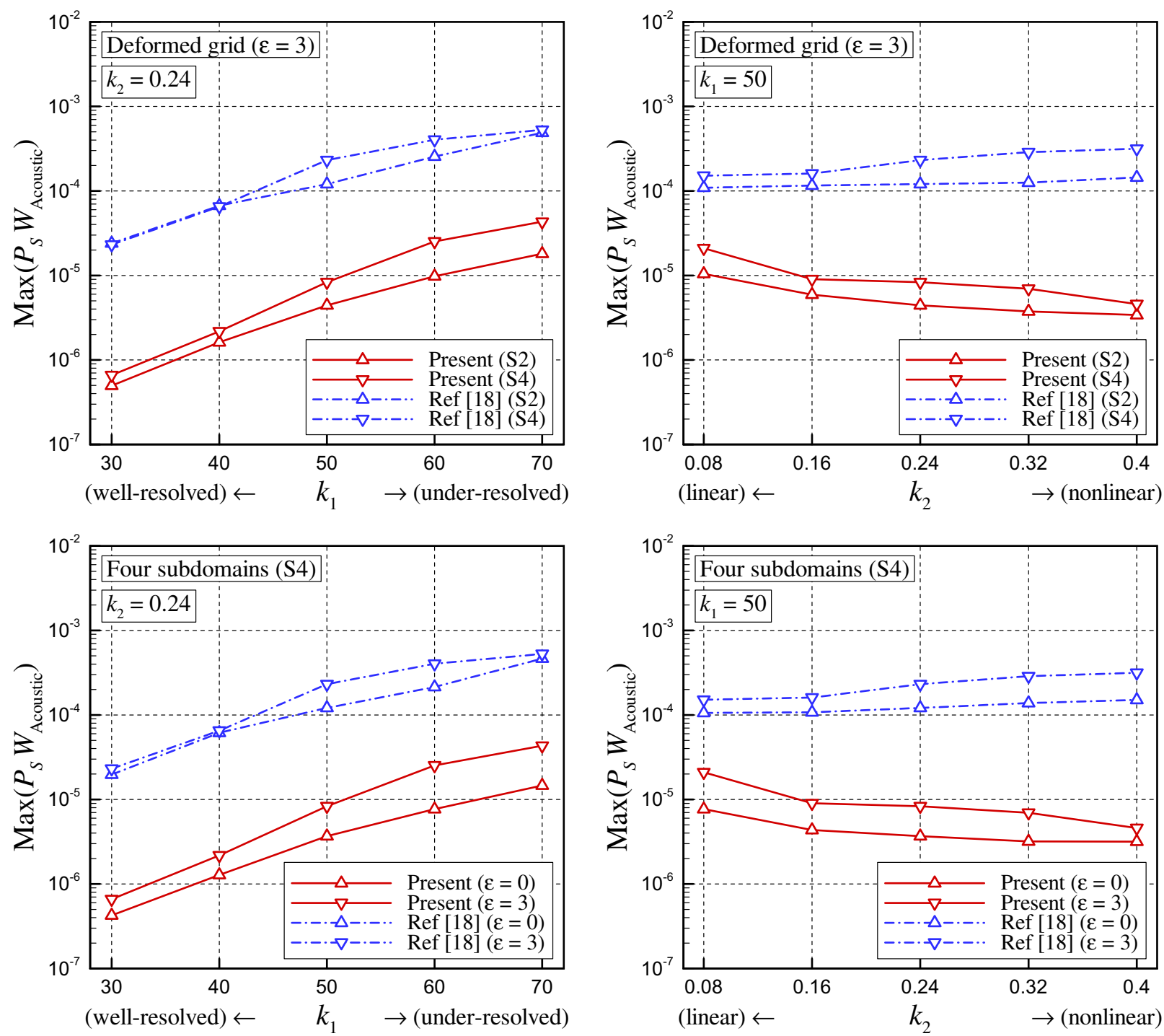

Fig. 16. Plots of largest values of $P_{S} W_{\text {Acoustic }}$ from Eq. (5.6) measured at $u_{\infty} t / L=0.25$ from the calculation of isentropic vortex convection with various parameters. S2 and S4 denote cases for two (split in streamwise direction) and four subdomains (split in lateral direction in addition to former).

\section{Table 6}

Parallel computing efficiencies calculated from aggregate CPU time elapsed per time step per grid point in two-dimensional isentropic vortex convection. S2 and S4 represent domains split into two and four subdomains, respectively, in the streamwise direction and additionally in the lateral direction.

\begin{tabular}{|c|c|c|c|c|c|}
\hline & \multirow[t]{2}{*}{ Single domain } & \multicolumn{2}{|c|}{ Present approach } & \multicolumn{2}{|c|}{ Ref. [18] } \\
\hline & & S2 & S4 & S2 & S4 \\
\hline Aggregate CPU time $(\mu \mathrm{s})$ & 0.7654 & 0.8002 & 0.8284 & 0.8806 & 0.9437 \\
\hline Relative efficiency & 1 & 0.9565 & 0.9240 & 0.8692 & 0.8111 \\
\hline Prediction - Eq. (3.18) & 1 & 0.9640 & 0.9305 & 0.9060 & 0.8282 \\
\hline
\end{tabular}

extra term $r_{c}$ that is a constant in the original Scully vortex model removes numerical singularity at the center of vortices. This paper suggests using a modified form of $r_{c}$ that diminishes after a certain distance from the vortex center in order to maintain uniform circulation in most of the computational domain, as follows:

$$
r_{c}=10^{-2\left(r_{m} / R\right)^{2}} \sigma L
$$

which reaches its maximum of $\sigma L$ (a fraction of $L$ ) at the center and reduces rapidly to $1 \%$ of it at $r_{m} / L=1$. For the present simulation, $\sigma=0.2$ is selected. The simulation is based on full nonlinear compressible Euler equations in a conservative form in generalized coordinates. 

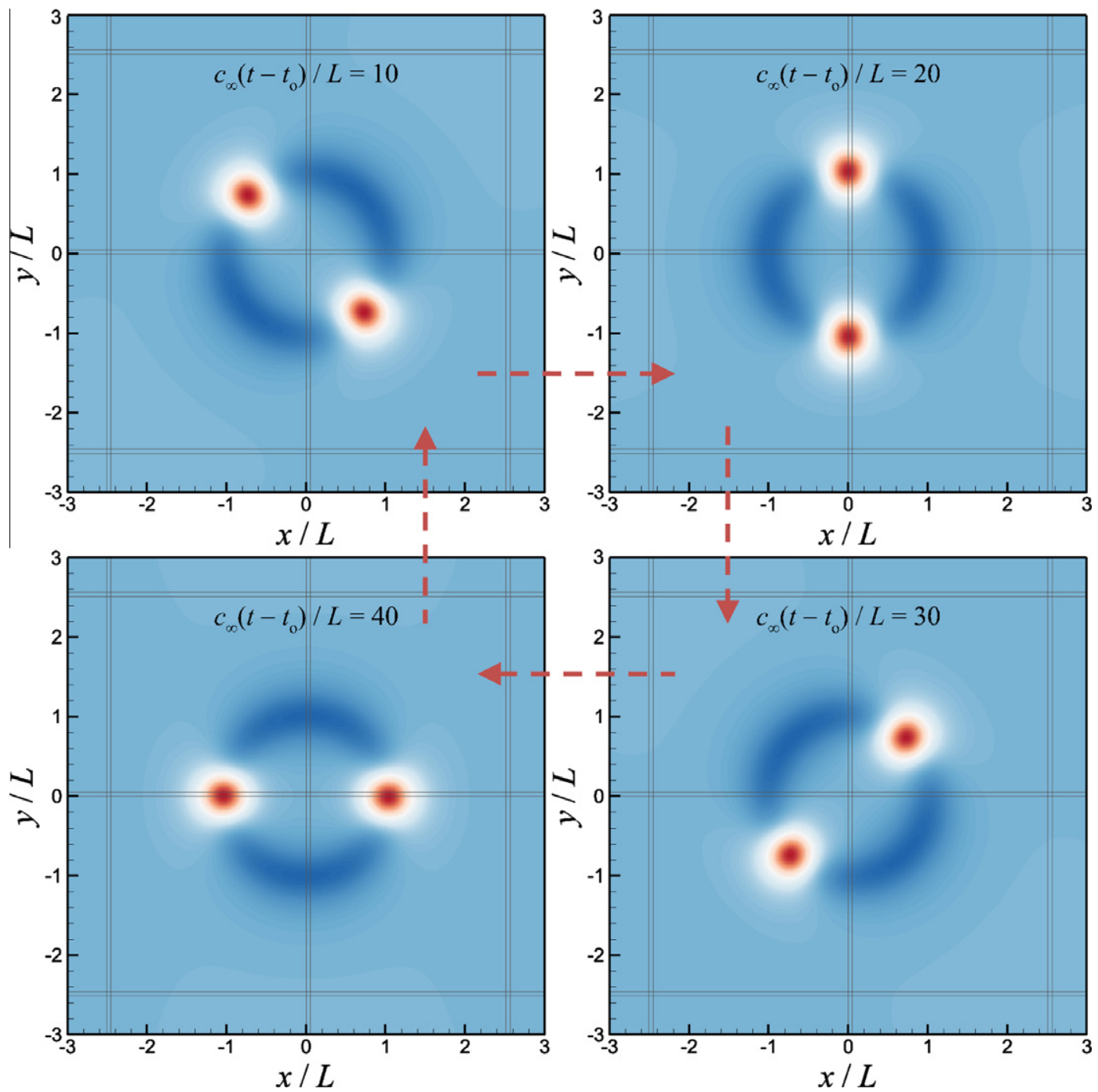

Fig. 17. Co-rotating vortex pair captured at every $1 / 8$ th of revolution $\left(t_{o}=t_{\max }-T_{o} / 2\right)$ : instantaneous plots of unsteady pressure normalized by ambient pressure $\left(p-p_{\text {mean }}\right) / p_{\infty}$. Contours run from -0.305 to 0.075 in 101 levels. Solid lines in the pictures denote subdomain boundaries ( 36 subdomains and processor-cores are used).

In an ideal potential flow that can be obtained if $\delta=r_{c}=0$ (point vortices) in Eq. (5.7), the period of co-rotation $\left(T_{o}\right)$ of the vortices can be related with the desired circulation as $\Gamma_{o} T_{o}=8 \pi^{2} L^{2}$. The non-ideal velocity field generated by introducing an artificial damping $\left(r_{c}\right)$ near the vortex cores may result in a different co-rotation speed of the vortices, which can be compensated by using the correction factor $\delta$. The present calculation is based on a co-rotation period $T_{o} c_{\infty} / L=80$ (by setting the desired circulation to $\left.\Gamma_{o} /\left(c_{\infty} L\right)=\pi^{2} / 10\right)$, which produces sound waves with the wavelength of $\lambda / L=40$. Attempting a few trial calculations, the correction factor of $\delta=0.059395$ has been found to meet the aimed co-rotation period. Given the flow conditions, an analytical solution derived in [21] predicts the induced acoustic pressure as follows:

$$
p_{a}(r, \theta, t)=-\frac{\rho_{\infty} \Gamma_{\mathrm{o}}^{4}}{64 \pi^{3} c_{\infty}^{2} L^{4}}\left[J_{2}\left(\frac{2 \Omega_{0} r}{c_{\infty}}\right) \sin \left(2 \theta+2 \Omega_{0} t\right)+Y_{2}\left(\frac{2 \Omega_{0} r}{c_{\infty}}\right) \cos \left(2 \theta+2 \Omega_{0} t\right)\right]
$$

where $J_{2}(z)$ and $Y_{2}(z)$ are the second-order Bessel functions of the first and second kind, respectively; $\Omega_{o}=2 \pi / T_{o}$ corresponds to the angular velocity of the co-rotation; and $(r, \theta)$ is the polar coordinates of the observer location from the center of the vortex pair.

Direct simulations of the co-rotating vortices and the induced sound waves are performed within a domain of $x \in[-340 L, 340 L]$ and $y \in[-340 L, 340 L]$. Stretched rectangular grid cells are used for this test and the total number of cells is $(N \times N)=(300 \times 300)$. The domain is split into 36 subdomains with an equal number of cells. Each subdomain is allocated to one processor-core (36 cores in total). The vortex pair is positioned at the center of the domain so that each vortex passes 

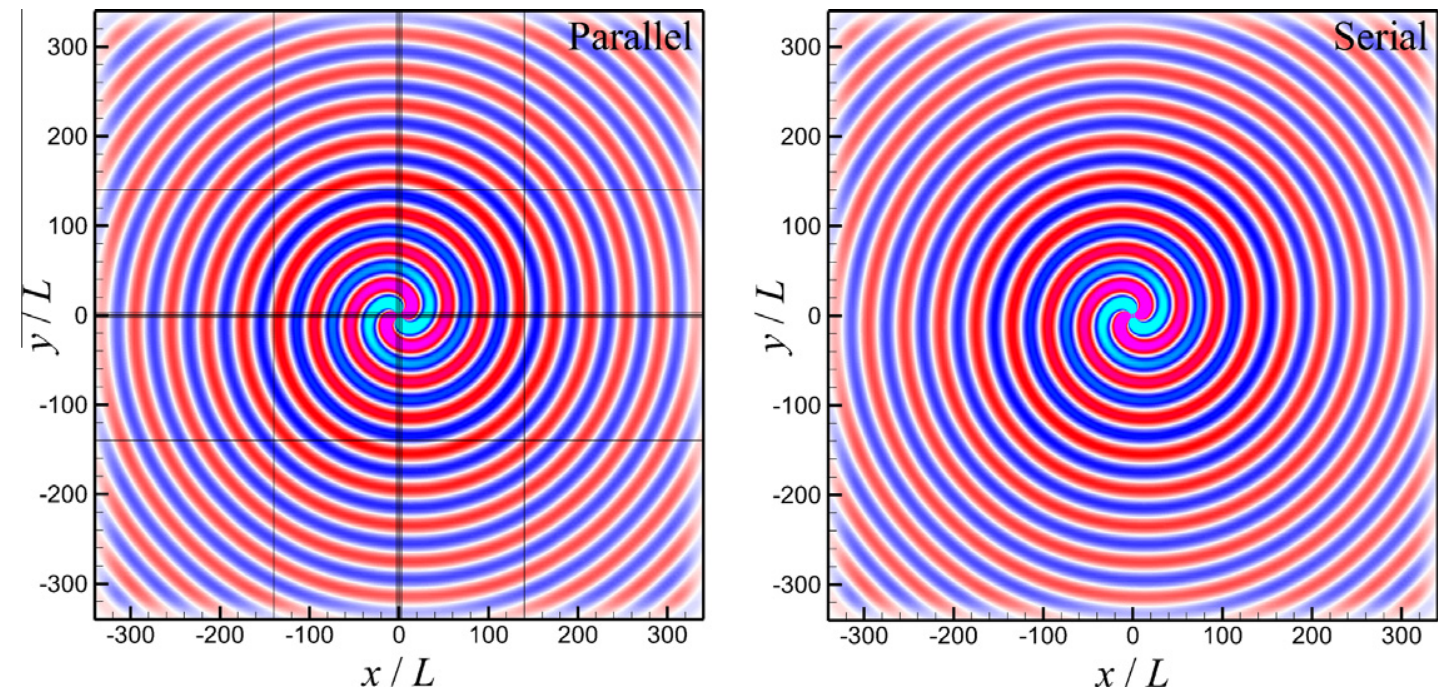

Fig. 18. Quadrupole sound field generated by a co-rotating vortex pair: instantaneous plots of acoustic pressure normalized by ambient pressure $\left(p-p_{\text {mean }}\right) / p_{\infty}$ obtained at the end of calculation. Contours run from $-2 \times 10^{-4}$ to $2 \times 10^{-4}$ in 101 levels. Solid lines in the left picture denote subdomain boundaries (36 subdomains and processor-cores are used).
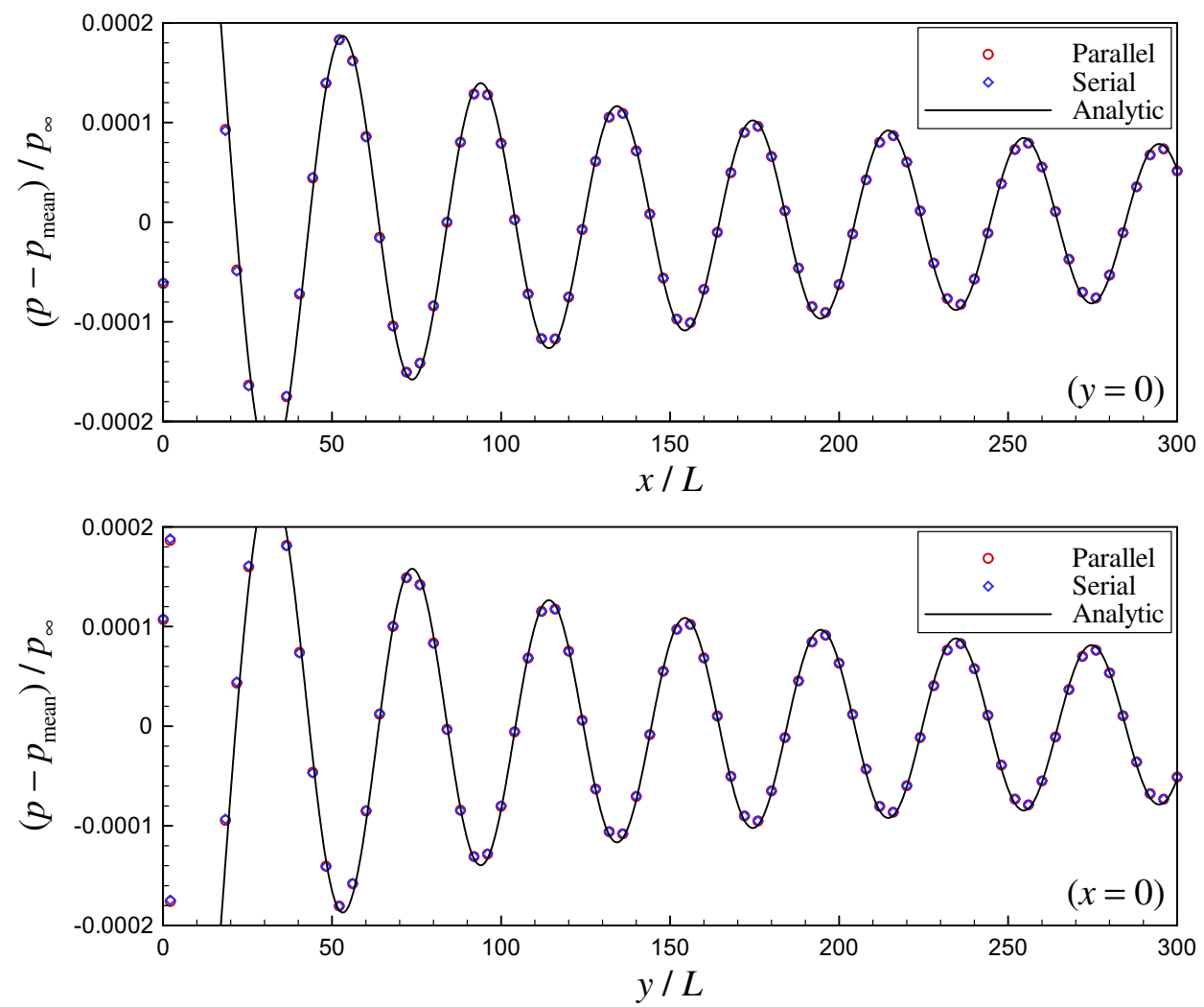

Fig. 19. Instantaneous profiles of acoustic pressure $\left(p-p_{\text {mean }}\right) / p_{\infty}$ from the simulation of a co-rotating vortex pair: measured along $x$-axis (top) and $y$-axis (bottom) of the domain.

through four different subdomain boundaries per revolution. Fine cells with the size of $\Delta x / L=\Delta y / L=0.05$ are located within a region of $x \in[-2 L, 2 L]$ and $y \in[-2 L, 2 L]$ surrounding the vortex pair, and the cell size grows exponentially up to $\Delta x / L=\Delta y /$ $L=4$ at the far corners of the domain. The present grid covers each vortex with around 16 cells (comparable to the moderately resolved case of $k_{1}=50$ in the earlier vortex convection test) and the sound wavelength with 10 cells at the far boundary. Characteristic boundary conditions [20] with the time derivatives of all the incoming components set to zero are 


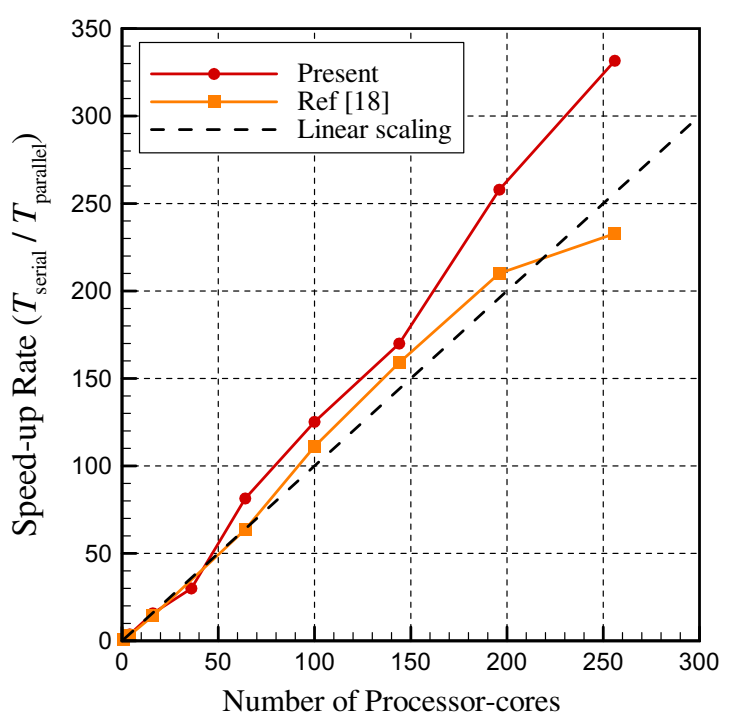

Fig. 20. Speed-up rates of parallel computing in the calculation of a co-rotating vortex pair.

imposed at the far boundary to avoid spurious wave reflections. A sponge layer [22] surrounding the far boundary with the thickness of $40 L$ (equal to the sound wavelength) is also used in order to ensure the reflection-free condition. The time step size is fixed at $c_{\infty} \Delta t / L=0.01725$ that corresponds to the CFL number of $0.9 \pm 0.005$ (variable in time). The calculations run until $c_{\infty} t / L=597.3366$ (rather than 600.0 to compensate a phase shift during initial transition) by which each vortex has circled 15 revolutions (crossing the subdomain boundaries 60 times) and returned to its initial position.

The result of calculation is shown in Figs. 17 and 18 which visualize the near-field unsteady pressure distribution and the far-field quadrupole sound radiation generated by the co-rotating vortex pair. It is demonstrated in Fig. 17 that the vortex pair leaves no trace of hydrodynamic artifacts after numerous events of crossing the subdomain boundaries. The parallel solution obtained by using 36 processor-cores is compared with the serial counterpart in Fig. 18 where no visible difference is identified even in the fully acoustic contour levels. The present vortex condition results in a core pressure drop up to $41 \%$ $\left(p_{\infty}-p_{\min }=0.41 p_{\infty}\right)$. Based on the results obtained in Section 5.2 (Fig. 16), the level of acoustic artifacts for the moderately resolved vortices may be estimated to reach $5 \times 10^{-6}\left(p_{\infty}-p_{\min }\right)=2 \times 10^{-6} p_{\infty}$ in the vicinity of the vortices, and it will become weaker as it radiates away from the center with the factor of $1 / r$. This means that the level of acoustic artifacts at the far field is as low as $10^{-8} p_{\infty}$ that hardly affects the genuine acoustic solution. The acoustic pressure data are compared with the analytic solution given by Eq. (5.9) in Fig. 19. The present data show excellent agreement with the analytic solution apart from reasonable discrepancies in the near to mid field $(r / L<10)$ where hydrodynamic fluctuations are predominant. More importantly, it is demonstrated that there exists no meaningful difference between the parallel and the serial data throughout the domain even in the fine acoustic pressure range.

The calculation is also performed in a massively parallel computing setup employing up to 256 processor-cores in order to examine the scalability of the new parallel computing approach. The task was carried out in IRIDIS3 cluster at the University of Southampton which is one of the largest and fastest university-based supercomputers at present. For this test, the corotating vortex pair was recalculated by using an increased number of cells $(N \times N)=(1120 \times 1120)$ and the job was evenly distributed over $k^{2}$ processor-cores where $k=(2,4,6,8,10,12,14,16)$. Fig. 20 shows that the present parallel computing approach provides superior speed-up rates to those of the old approach in [18], particularly when 150 or more processor-cores are used. It is also observed that the present setup exhibits super-linear scalability at large numbers of processor-cores. Note that none of the pre-/post-processing time is involved in the results of Fig. 20. The super-linear scalability is perhaps attributed to the fact that the relative amount of calculations performed within L1/L2 CPU Cache (which is much faster than RAM/ DRAM) increases as the size of subdomains becomes smaller. This extra speedup might have compensated the internode communication overhead. In the meantime, it seems that the iterative filtering method used in [18] fails to fully take advan-

Table 7

Comparison of parallel artifacts in pressure measured in an $L_{2}$-norm basis in the calculation of a co-rotating vortex pair distributed over 36 processor-cores for three different numbers of cells used.

\begin{tabular}{lll}
\hline Number of cells used: $(N \times N)$ & {$\left[\sum_{j=0}^{N} \sum_{i=0}^{N}\left(p_{\text {parallel }}-p_{\text {serial }}\right)^{2}\right]^{1 / 2} /\left[p_{\infty}(N+1)\right]$} \\
\cline { 2 - 3 } & Present approach & Ref. [18] \\
\hline$(150 \times 150)$ & $6.5550 \mathrm{e}-3$ & $4.8860 \mathrm{e}-2$ \\
$(300 \times 300)$ & $9.9624 \mathrm{e}-5$ & $1.1434 \mathrm{e}-3$ \\
$(600 \times 600)$ & $2.4374 \mathrm{e}-6$ & $3.8284 \mathrm{e}-5$ \\
\hline
\end{tabular}


tage of the beneficial effects due to the increased amount of calculation and internode communication. Finally, it is re-confirmed in Table 7 that the present approach generates an order of magnitude smaller parallel artifacts than those of [18] in the last benchmark test case as well.

\section{Concluding remarks}

The proposed BAA (boundary approximation approach) has successfully led to the development of new SB (subdomain boundary) compact finite-difference schemes and filters for parallel computing purposes. The new SB compact schemes and filters require three halo terms for a conventional message passing process, which demands the same cost of inter-node communication as that of standard explicit schemes and filters based on seven-point stencils. Non-uniformity in resolution characteristics across subdomain boundaries, which was the major drawback in the earlier works of BAA, has been substantially reduced by a factor of 10 (or more if required) in this paper. This is achieved by implementing a novel linear-algebraic transformation technique to obtain quasi-disjoint pentadiagonal matrix systems, which leads to deriving the SB compact schemes and filters with variable stencil sizes. It is found that $M=11$ and $M_{F}=8$ are optimal stencil sizes for the present SB compact schemes and filters to keep the resolution non-uniformity below $3 \%$ and the combined parallel efficiency over $92 \%$ for a reasonably sized subdomain $\left(N_{S}=100\right)$. Strictly artifact-free conditions may also be achieved if necessary by choosing $M=M_{F}=19$ at an extra computational cost. This approach may be used for various compact schemes/filters and other banded matrix solver systems in general to achieve both high accuracy and efficiency in parallel computing environments.

The present benchmark tests evidence that the enhanced uniformity in resolution characteristics does improve the accuracy of parallel computing and reduce the discrepancy between the parallel and the serial solution by a factor of 10 to 100 consistently compared with the latest published results [18]. The overall accuracy of the proposed SB compact schemes and filters are proved to be of fourth- and fifth-order, respectively. The maximum levels of hydrodynamic and acoustic artifacts (normalized by the major hydrodynamic perturbation) in the proposed approach are estimated to be in the order of $10^{-5}$ and $10^{-6}$ (or lower), respectively, at a moderate to sufficient grid resolution. This means that the number of valid digits in numerical data will be fairly sufficient for most aeroacoustic simulations. This claim is also supported by the calculation of co-rotating vortices that represent the weakest source of sound (quadrupole) predominant in low-speed free shear flows. It is shown that the level of acoustic artifacts generated after numerous repetitions of collisions between the vortices and subdomain boundaries make no meaningful impact on the genuine acoustic solution. Also, the present parallel computing setup exhibits super-linear scalability when a large number of processor-cores are used. The proposed approach with the new SB compact schemes and filters may be used for high-fidelity parallel computing of various aeroacoustic problems.

\section{Appendix A}

The filter coefficients in Eqs. (2.3) and (2.4) can be determined by selecting an appropriate cut-off wavenumber $(\pi /$ $\left.2 \leqslant \kappa_{C} \leqslant \pi\right)$ and a boundary weighting factor $\left(0 \leqslant \varepsilon_{F} \ll 1\right)$ in accordance with the finite-difference schemes used. The coefficients are determined by the following formulas given by [12]:

$$
\begin{aligned}
& \alpha_{F}=-\left[30 \cos \left(\kappa_{C}\right)+2 \cos \left(3 \kappa_{C}\right)\right] / A_{F}\left(\kappa_{C}\right) \\
& \left.\begin{array}{l}
\beta_{F}=\left[18+9 \cos \left(\kappa_{C}\right)+6 \cos \left(2 \kappa_{C}\right)-\cos \left(3 \kappa_{C}\right)\right] /\left[2 A_{F}\left(\kappa_{C}\right)\right] \\
a_{F 1}=30 \cos ^{4}\left(\kappa_{C} / 2\right) / A_{F}\left(\kappa_{C}\right), \quad a_{F 2}=-2 a_{F 1} / 5, \quad a_{F 2}=a_{F 1} / 15 \\
A_{F}\left(\kappa_{C}\right)=30-5 \cos \left(\kappa_{C}\right)+10 \cos \left(2 \kappa_{C}\right)-3 \cos \left(3 \kappa_{C}\right)
\end{array}\right\} \quad \text { for } 3 \leqslant i \leqslant N-3, \\
& \left.\begin{array}{l}
\gamma_{F 20}=\dot{\beta}_{F}, \quad \gamma_{F 21}=\dot{\alpha}_{F}, \quad \gamma_{F 23}=\dot{\alpha}_{F}, \quad \gamma_{F 24}=\dot{\beta}_{F} \\
b_{F 20}=\dot{a}_{F 2}+5 \dot{a}_{F 3}, \quad b_{F 21}=\dot{a}_{F 1}-10 \dot{a}_{F 3}, \quad b_{F 23}=\dot{a}_{F 1}-5 \dot{a}_{F 3}, \quad b_{F 24}=\dot{a}_{F 2}+\dot{a}_{F 3}, \quad b_{F 25}=\dot{a}_{F 3}
\end{array}\right\} \quad \text { for } i=\left\{\begin{array}{l}
2, \\
N-2,
\end{array}\right. \\
& \gamma_{F 10}=\left[10 \ddot{\beta}_{F}^{2}\left(8 \ddot{\beta}_{F}-1\right)+\left(1+4 \ddot{\beta}_{F}+81 \ddot{\beta}_{F}^{2}\right) \ddot{\alpha}_{F}+5\left(1+8 \ddot{\beta}_{F}\right) \ddot{\alpha}_{F}^{2}+9 \ddot{\alpha}_{F}^{3}\right] / B_{F} \\
& \gamma_{F 12}=\left[\ddot{\alpha}_{F}\left(1+5 \ddot{\alpha}_{F}+9 \ddot{\alpha}_{F}^{2}\right)+\ddot{\alpha}_{F}\left(5+36 \ddot{\alpha}_{F}\right) \ddot{\beta}_{F}+\left(55 \ddot{\alpha}_{F}-1\right) \ddot{\beta}_{F}^{2}+10 \ddot{\beta}_{F}^{3}\right] / B_{F} \\
& \gamma_{F 13}=\ddot{\beta}_{F}\left[1+5 \ddot{\alpha}_{F}+9 \ddot{\alpha}_{F}^{2}+5\left(1+7 \ddot{\alpha}_{F}\right) \ddot{\beta}_{F}+50 \ddot{\beta}_{F}^{2}\right] / B_{F} \\
& \text { for } i=\left\{\begin{array}{l}
1, \\
N-1
\end{array}\right. \\
& B_{F}=\left(1-\ddot{\beta}_{F}\right)\left(1+6 \ddot{\beta}_{F}+60 \ddot{\beta}_{F}^{2}\right)+\left(5+35 \ddot{\beta}_{F}-29 \ddot{\beta}_{F}^{2}\right) \ddot{\alpha}_{F}+\left(9-5 \ddot{\beta}_{F}\right) \ddot{\alpha}_{F}^{2} \\
& \gamma_{F 01}=\left[\dddot{\alpha}_{F}\left(1+\dddot{\alpha}_{F}\right)\left(1+4 \dddot{\alpha}_{F}\right)+2 \dddot{\alpha}_{F}\left(7+3 \dddot{\alpha}_{F}\right) \dddot{\beta}_{F}+24\left(1-\dddot{\alpha}_{F}\right) \dddot{\beta}_{F}^{2}-80 \dddot{\beta}_{F}^{3}\right] / C_{F} \\
& \gamma_{F 02}=\left[\dddot{\alpha}_{F}^{3}+\left(1+3 \dddot{\alpha}_{F}+14 \dddot{\alpha}_{F}^{2}\right) \dddot{\beta}_{F}+46 \dddot{\alpha}_{F} \dddot{\beta}_{F}^{2}+60 \dddot{\beta}_{F}^{3}\right] / C_{F} \\
& \left.C_{F}=1+\dddot{\beta}_{F}\left(5+4 \dddot{\beta}_{F}+60 \dddot{\beta}_{F}^{2}\right)+5\left(1+3 \dddot{\beta}_{F}+10 \dddot{\beta}_{F}^{2}\right) \dddot{\alpha}_{F}+2\left(4+11 \dddot{\beta}_{F}\right) \dddot{\alpha}_{F}^{2}+5 \ddot{\alpha}_{F}^{3}\right) \\
& \text { for } i=\left\{\begin{array}{l}
0, \\
N,
\end{array}\right.
\end{aligned}
$$


where $\kappa_{C}$ is the cut-off wavenumber normalized by grid space $\left(\kappa_{C}=k_{C} \Delta x\right)$. The dots “.”, “..” and “...” in Eqs. (A.2)-(A.4) denote boundary filter coefficients calculated from weighted cut-off wavenumbers for enhanced stability at the boundaries [12]. The weighted boundary cut-off wavenumbers are given by

$$
\left(\dot{\kappa}_{C}, \ddot{\kappa}_{C}, \dddot{\kappa}_{C}\right)=\kappa_{C}\left[1-\varepsilon_{F} \sin ^{2}(\pi / 6, \pi / 3, \pi / 2)\right]
$$

which provides a linearly decreasing cut-off wavenumber from $\kappa_{C}$ to $\kappa_{C}\left(1-\varepsilon_{F}\right)$ across three boundary cells. In the present work, $\kappa_{C}=0.88 \pi$ and $\varepsilon_{F}=0.25$ are used as suggested in [12]. The use of boundary weighting is limited to physical boundaries only. The derivation of SB filters in Section 3 is based on zero weighting $\left(\varepsilon_{F}=0\right)$ in order to maintain the uniformity of cut-off wavenumbers across subdomain boundaries.

\section{Appendix B}

(See Tables B1 and B2).

Table B1

Coefficients for proposed SB compact finite-difference with an efficient stencil size $(M=11)$ for $3 \%$ of Fourier error criterion $(\Phi<0.03)$ from Eq. (4.10).

\begin{tabular}{|c|c|c|}
\hline & $i=0$ & $i=1$ \\
\hline$c_{i 11}^{*}$ & $8.992896791505667 e-4$ & $1.8805388760373756 \mathrm{e}-4$ \\
\hline$c_{i 10}^{*}$ & $-4.986658746868655 e-3$ & $-1.0428064609290105 e-3$ \\
\hline$c_{i 9}^{*}$ & 0.012786121380103266 & $2.674020687502082 \mathrm{e}-3$ \\
\hline$c_{i 8}^{*}$ & -0.022191498180909847 & $-4.642009649365245 \mathrm{e}-3$ \\
\hline$c_{i 7}^{*}$ & 0.032927991618090485 & $6.892064579400338 \mathrm{e}-3$ \\
\hline$c_{i 6}^{*}$ & -0.04657661323376615 & $-9.765163647505088 \mathrm{e}-3$ \\
\hline$c_{i 5}^{*}$ & 0.06440194430816426 & 0.013564972483922279 \\
\hline$c_{i 4}^{*}$ & -0.08928335697480955 & -0.019041949765658123 \\
\hline$c_{i 3}^{*}$ & 0.1269011204772041 & 0.027944770534486005 \\
\hline$c_{i 2}^{*}$ & -0.1899431297805324 & -0.045046905179487715 \\
\hline$c_{i 1}^{*}$ & 0.31949304859383254 & 0.08695470058860919 \\
\hline$c_{i 0}^{*}$ & -0.736992740887527 & -0.2584771941406698 \\
\hline$c_{i 0}$ & -0.5336813436634584 & -0.737493882305206 \\
\hline$c_{i 1}$ & 0.8929327012121225 & 0.04533056718142581 \\
\hline$c_{i 2}$ & 0.10470378604054187 & 0.6148876130753849 \\
\hline$c_{i 3}$ & 0.11170443847314782 & 0.27789867343514313 \\
\hline$c_{i 4}$ & -0.07406941462165517 & $-6.5575218744913025 e-3$ \\
\hline$c_{i 5}$ & 0.05356599552451824 & $9.914395938076442 \mathrm{e}-3$ \\
\hline$c_{i 6}$ & -0.038776573996292044 & $-7.179137445315696 \mathrm{e}-3$ \\
\hline$c_{i 7}$ & 0.02742301575120666 & $5.077657345955812 \mathrm{e}-3$ \\
\hline$c_{i 8}$ & -0.018483768633962283 & $-3.4225923067379947 e-3$ \\
\hline$c_{i 9}$ & 0.010650298015870585 & $1.9721149221814116 \mathrm{e}-3$ \\
\hline$c_{i 10}$ & $-4.153737783508027 e-3$ & $-7.691508381817702 \mathrm{e}-4$ \\
\hline$c_{i 11}$ & $7.490854293364865 e-4$ & $1.3870895385643608 \mathrm{e}-4$ \\
\hline
\end{tabular}

Table B2

Coefficients for proposed SB compact filters with an efficient stencil size $\left(M_{F}=8\right)$ for $3 \%$ of Fourier error criterion $\left(\Phi_{F}<0.03\right)$ from Eq. (4.10).

\begin{tabular}{lll}
\hline & $i=0$ & \\
\hline$c_{F i 8}^{*}$ & 0.0004080671697386847 & 0.00015993165225968903 \\
$c_{F i 7}^{*}$ & -0.002717077311573497 & -0.0010578318951607495 \\
$c_{F i 6}^{*}$ & 0.008480664890910771 & 0.003267326218563917 \\
$c_{F i 5}^{*}$ & -0.017334702567069534 & -0.006573193658529932 \\
$c_{F i 4}^{*}$ & 0.02779543950845714 & 0.010312204999438773 \\
$c_{F i 3}^{*}$ & -0.03912303782296065 & -0.014132468145873005 \\
$c_{F i 2}^{*}$ & 0.050670406049655274 & 0.017740605419707154 \\
$c_{F i 1}^{*}$ & -0.060616551731385186 & -0.020410018015663934 \\
$c_{F i 0}^{*}$ & 0.06618452008562463 & 0.021181984429966882 \\
$c_{F i 0}^{*}$ & -0.0651664813842433 & -0.019704550203409617 \\
$C_{F i 1}$ & 0.057834156017972066 & 0.01678572683413678 \\
$c_{F i 2}$ & -0.04690486952135603 & -0.013552985219781221 \\
$c_{F i 3}$ & 0.03532589000463139 & 0.01041393174226837 \\
$C_{F i 4}$ & -0.02463082407109682 & -0.007354410488865658 \\
$c_{F i 5}$ & 0.015138255674794043 & 0.004501738899644245 \\
$c_{F i 6}$ & -0.007325228325767596 & -0.002158677210552717 \\
$c_{F i 7}$ & 0.002329579902198938 & 0.0006822496763485686 \\
$C_{F i 8}$ & -0.0003482065685303191 & -0.00010156503449754444 \\
\hline & & \\
\hline
\end{tabular}




\section{References}

[1] S.K. Lele, Compact finite difference schemes with spectral-like resolution, J. Comput. Phys. 103 (1) (1992) 16.

[2] M.H. Carpenter, D. Gottlieb, S. Abarbanel, The stability of numerical boundary treatments for compact high-order finite-difference schemes, J. Comput. Phys. 108 (2) (1993) 272.

[3] J.W. Kim, D.J. Lee, Optimized compact finite difference schemes with maximum resolution, AIAA J. 34 (5) (1996) 887.

[4] M.A. Tolstykh, On the performance of methods with third- and fifth-order compact upwinding differencing, J. Comput. Phys. 140 (2) (1998) 205.

[5] J.A. Ekaterinaris, Implicit, high-resolution, compact schemes for gas dynamics and aeroacoustics, J. Comput. Phys. 156 (2) (1999) 272.

[6] R. Hixon, Prefactored small-stencil compact schemes, J. Comput. Phys. 165 (2) (2000) 522.

[7] D.V. Gaitonde, M.R. Visbal, Pade-type higher-order boundary filters for the Navier-Stokes equations, AIAA J. 38 (11) (2000) 2103.

[8] M.R. Visbal, D.V. Gaitonde, On the use of higher-order finite-difference schemes on curvilinear and deforming meshes, J. Comput. Phys. 181 (1) (2002) 155.

[9] S. Nagarajan, S.K. Lele, J.H. Ferziger, A robust high-order compact method for large eddy simulation, J. Comput. Phys. 191 (2) (2003) 392.

[10] S.E. Sherer, J.N. Scott, High-order compact finite-difference methods on general overset grids, J. Comput. Phys. 210 (2) (2005) 459.

[11] J.W. Kim, Optimised boundary compact finite difference schemes for computational aeroacoustics, J. Comput. Phys. 225 (1) (2007) 995.

[12] J.W. Kim, High-order compact filters with variable cut-off wavenumber and stable boundary treatment, Comput. Fluids 39 (7) (2010) 1168.

[13] J. Hofhaus, E.F. Van de Velde, Alternating-direction line-relaxation methods on multicomputers, SIAM J. Sci. Comput. 17 (2) (1996) 454.

[14] A. Povitsky, P.J. Morris, A higher-order compact method in space and time based on parallel implementation of the Thomas algorithm, J. Comput. Phys. 161 (1) (2000) 182

[15] X.H. Sun, S. Moitra, A fast parallel tridiagonal algorithm for a class of CFD applications, NASA Technical Paper $3585,1996$.

[16] E. Polizzi, A.H. Sameh, SPIKE: a parallel environment for solving banded linear systems, Comput. Fluids 36 (1) (2007) 113.

[17] T.K. Sengupta, A. Dipankar, A.K. Rao, A new compact scheme for parallel computing using domain decomposition, J. Comput. Phys. 220 (2) (2007) 654.

[18] J.W. Kim, R.D. Sandberg, Efficient parallel computing with a compact finite difference scheme, Comput. Fluids 58 (1) (2012) 70.

[19] C.K. W Tam, J.C. Webb, Dispersion-relation-preserving finite difference schemes for computational aeroacoustics, J. Comput. Phys. 107 (2) (1993) 262.

[20] J.W. Kim, D.J. Lee, Generalized characteristic boundary conditions for computational aeroacoustics, part 2, AIAA J. 42 (1) (2004) 47.

[21] B.E. Mitchell, S.K. Lele, P. Moin, Direct computation of the sound from a compressible co-rotating vortex pair, J. Fluid Mech. 285 (1) (1995) 181.

[22] J.W. Kim, A.S.H. Lau, N.D. Sandham, Proposed boundary conditions for gust-airfoil interaction noise, AIAA J. 48 (11) (2010) 2705. 